IZA DP No. 9619

Working Time Reductions at the End of the Career:

Do They Prolong the Time Spent in Employment?

Andrea Albanese

Bart Cockx

Yannick Thuy

December 2015 


\title{
Working Time Reductions at the End of the Career: Do They Prolong the Time Spent in Employment?
}

\author{
Andrea Albanese \\ SHERPPA, Ghent University \\ Bart Cockx \\ SHERPPA, Ghent University, IRES, Université Catholique de Louvain, \\ CESifo and IZA \\ Yannick Thuy \\ SHERPPA, Ghent University

\section{Discussion Paper No. 9619 \\ December 2015} \\ IZA \\ P.O. Box 7240 \\ 53072 Bonn \\ Germany \\ Phone: +49-228-3894-0 \\ Fax: +49-228-3894-180 \\ E-mail: iza@iza.org
}

Any opinions expressed here are those of the author(s) and not those of IZA. Research published in this series may include views on policy, but the institute itself takes no institutional policy positions. The IZA research network is committed to the IZA Guiding Principles of Research Integrity.

The Institute for the Study of Labor (IZA) in Bonn is a local and virtual international research center and a place of communication between science, politics and business. IZA is an independent nonprofit organization supported by Deutsche Post Foundation. The center is associated with the University of Bonn and offers a stimulating research environment through its international network, workshops and conferences, data service, project support, research visits and doctoral program. IZA engages in (i) original and internationally competitive research in all fields of labor economics, (ii) development of policy concepts, and (iii) dissemination of research results and concepts to the interested public.

IZA Discussion Papers often represent preliminary work and are circulated to encourage discussion. Citation of such a paper should account for its provisional character. A revised version may be available directly from the author. 
IZA Discussion Paper No. 9619

December 2015

\section{ABSTRACT \\ Working Time Reductions at the End of the Career: Do They Prolong the Time Spent in Employment?*}

In this paper we study the effects on the survival rate in employment of a scheme that facilitates gradual retirement through working time reductions. We use information on the entire labour market career and other observables to control for selection and take dynamic treatment assignment into account. We also estimate a competing risks model considering different (possibly selective) pathways to early retirement. We find that participation in the scheme initially prolongs employment, as participants keep accumulating full pension rights. However, as participants become eligible for early retirement subsequently, these larger financial incentives induce them to leave the labour force prematurely. These adverse incentives are stronger for individuals who reduce their working time most. After two (four) years for men (women), the positive effects reverse. The more favourable effect for women is likely a consequence of their lower opportunities to enter early retirement. The gradual retirement scheme fails the cost-benefit test.

JEL Classification: J14, C22, J18, J22

Keywords: $\quad$ part-time work, older workers, Inverse Probability Weighting, dynamic selection into treatment, endogenous sampling

Corresponding author:

Bart Cockx

Ghent University

Sint-Pietersplein 6

B-9000 Gent

Belgium

E-mail: Bart.Cockx@ugent.be

\footnotetext{
"We acknowledge financial support for this research project from the programme "Society and Future" of the Belgian Science Policy (contract $\mathrm{n}^{\circ} \mathrm{TA} / 00 / 044$ ) and from the special Research Fund of Ghent University for providing a scholarship to Andrea Albanese (code 01SF3612). We thank the Crossroads Bank for Social Security for the delivery of the data (report nr. 12/080 of the Sectoral Commission of Social Security and Health, department "Social Security"). We are grateful to Lorenzo Cappellari, Michael Lechner, Marco Leonardi, Matteo Picchio and Bruno Van der Linden for the useful comments and discussions. We also thank participants to the Spring Meeting of Young Economists and the Belgian Day for Labour Economists for their valuable input.
} 


\section{Introduction}

Population ageing puts enormous pressure on Social Security provisions in many developed countries. One of the main factors involved in this is the low labour force participation of older workers, and in particular of older women. In EU-15 countries in 2013, the labour force participation rates of workers aged 55-64 was 29 percentage points below that of workers aged 25-54 (OECD, 2015a). This difference was even more pronounced for Belgium, the country of analysis in this paper, where the participation rate of the older age group is 41 percentage points lower than the younger one. Low labour market participation at older ages is in many developed countries in part caused by early retirement. Even though in many EU15 countries the average legal retirement age is around 65, people still tend to retire two and a half years earlier (OECD, 2015b). ${ }^{1}$ In Belgium workers are observed to retire particularly early, with an average effective retirement age below 60 since the late 1980's.

A key strategy to cope with the challenge induced by population ageing is to prolong the working career and to increase the activity rates of older workers. However, simply continuing to work full-time at an older age might increase the risk of dropping out from the labour force due to declining health or to taking up care obligations (Gielen, 2009; Van Looy et al., 2014). It has been argued that workers might stay longer in the labour force if they could gradually reduce their working time at the end of their career (e.g. Schmid, 1998).

Several EU countries have put gradual retirement schemes in place for older workers. ${ }^{2}$ These can take different forms. In Sweden, Finland and Denmark workers can reduce their working time and top up their income by prematurely drawing from their pension entitlements. In Belgium, Germany and Austria employees can reduce working time before entering into retirement and the government provides a subsidy to partially compensate for the income loss. In these countries employees can also choose the so called "block-model". This model concentrates the reduced working time in the years prior to retirement by taking a leave of absence, actually inducing retirement to be early instead of gradual. Finally, before the abolition in 2012, Dutch employees could 'save' time early in their career to reduce working time later on. In this paper we evaluate the Belgian scheme. We study whether this Time Credit (TC) scheme can lengthen the career of older private sector workers.

Evidence of the effectiveness of gradual retirement schemes based on counterfactual impact evaluations is scarce. Most research has focussed on studying the determinants of gradual retirement (e.g. Gustman and Steinmeier, 1984; Honig and Hanoch, 1985; Ruhm, 1990; Hutchens and GraceMartin, 2006; Hutchens, 2010; Kantarcı and van Soest, 2008, for a survey). Wadensjö (2006) uses the

\footnotetext{
${ }^{1}$ Average over the period 2007-2012.

${ }^{2}$ See Table A.1 in the Appendix A for an overview of such schemes in the countries mentioned in the text.
} 
counterfactual analysis of Sundén (1994) to determine the behaviour of part-time pensioners in Sweden in the counterfactual of no part-time pension and concludes that the scheme increased the number of hours worked in the economy. However, he underlines (on p. 27) that "the calculations are based on data which is not perfect for the task". Based on the conditional independence assumption (CIA), Graf et al. (2011) and Huber et al. (2015) estimate the effects of the Austrian, respectively German, gradual retirement scheme. Both studies find that gradual retirement reduces the likelihood of unemployment. However, this does not imply that these workers remain employed longer, because if the "block model" is chosen, the worker is officially employed without actually working. Although workers in the inactive phase of the block model are counted as employed, Graf et al. find that in Austria the effect on employment is eventually negative. Similarly, Huber et al. (2015) report for WestGerman participants in the scheme no significantly higher survival rate in employment. However, they do find significant positive employment effects for East-German participants and positive spill-over effects on younger workers. They attribute this differential finding to the difficult labour market conditions in East-Germany. Berg et al. (2015) also evaluate the German partial retirement scheme, but based on a difference-in-differences strategy. They find that participation in the scheme prolongs the working career for men, but less so in periods of more intensive use of the block model. Smaller and, in the period of more intensive use of the block model, even negative effects are reported for women. The authors do not consider differential effects for West- and East-Germany. Finally, Elsayed et al. (2015) use a stated preferences experiment based on vignettes to evaluate the effect of various hypothetical pension reforms in the Netherlands, among which the introduction of gradual retirement. This study finds that gradual retirement would induce workers to retire one year later on average, but also that total lifetime labour supply would still fall by 3.4 months.

In this paper we focus on the component of the Belgian TC scheme that targets workers older than fifty, because it entitles eligible individuals to the TC until retirement and can therefore be viewed as a gradual retirement scheme. We exploit very rich administrative data on labour market histories (for private sector employment from as early as 1957), sick leave, exit destinations out of employment, and some essential firm and household characteristics. Based on these data we can estimate the impact on the survival rate in employment until eight years after entry into TC. Similar to the studies of Graf et al. (2011) and Huber et al. (2015), we base identification on the CIA. We argue that this is a credible identification strategy because of the richness of the data and because the analysis is restricted to the eligible population, which is relatively homogeneous. To be eligible an individual should have worked at least five years full-time in the same private sector firm employing at least 11 employees and have accumulated at least 20 years of labour market experience. Moreover, even if information on important variables, such as ability, motivation and health histories, are missing, we 
follow Lechner et al. (2011) and Huber et al. (2015) by claiming that these unobservables are indirectly captured by conditioning on the very rich pre-treatment labour market histories. For instance, health problems should be reflected by gaps in the labour market experience or in lower earnings; Or, ability in the average earnings level.

We contribute to the literature in the following ways. First, because the block model actually resembles more an early retirement scheme than a gradual one, we restrict, in contrast to the aforementioned studies, the treatment to genuine part-time workers and consider participants in the block model as inactive if they stop working. Second, we investigate whether the treatment effect depends on the extent of working time reduction $-20 \%$ or $50 \%$. Third, we explicitly study the interaction between the impact of gradual retirement on the timing of withdrawal from the labour market and the supply incentives to exit the labour market via early retirement schemes. In particular, we provide suggestive evidence that the effectiveness of gradual retirement to prolong the labour market career depends on how participation in the TC scheme affects the level of the allowances in early retirement schemes. TC-beneficiaries keep on accumulating statutory pension rights of full-time workers, so that the relatively higher replacement income induces them to enter early statutory retirement more quickly than in the absence of TC. By contrast, TC-beneficiaries are less inclined to leave the labour force through alternative early retirement schemes, since the allowances in these schemes are less generous.

Fourth, to the best of our knowledge, we are the first to provide (partial) evidence on a health outcome by analysing the effect of gradual retirement on the incidence of sick leave during employment. Furthermore, we propose a cost-benefit analysis in which we estimate, based on the $\mathrm{CIA}$ and using the administrative data, both the net budgetary costs for the state and the net welfare gains (or costs) for society. Finally, from a methodological perspective, we explicitly take into account that the TC scheme is not entered at a fixed moment, but can happen at any time. Sianesi (2004), Fredriksson and Johansson (2008) and Crépon et al. (2009) have shown that in case of such dynamic assignment into treatment, methods based on the CIA that assume that the treatment assignment is static are biased. They propose propensity score matching methods that take this dynamic assignment explicitly into account. Vikström (2014) built on these findings to suggest a method that takes into account that the right censoring induced by transitions into treatment may depend on observables and, hence, be selective, a problem that was overlooked in the literature. We use this estimator, but adjust it to consider that, for reasons explained in Section 4, our analysis is based on an endogenously stratified sample. It is well known that in this case consistent estimation requires appropriate weighing of the 
data (Manski and Lerman, 1977). ${ }^{3}$ Such adjustment is straightforward in Vikström's estimator, as it is implemented as an inverse probability weighting (IPW) estimator (Horvitz and Thompson, 1952; Hirano et al., 2003): it merely consists in an extra weighing of the data to take the endogenous sampling into account.

Our findings can be summarized as follows. We estimate a positive short run effect of the TC on the survival rate in employment. This effect becomes negative after two (four) years for men (women). Qualitatively these effects are therefore similar to those found by Graf et al. (2011) for Austria. The negative effect is essentially explained by the fact that TC beneficiaries who enter statutory early retirement remain entitled to the level of pension benefits of a full-time worker, which makes early retirement relatively more attractive for those workers. Once we right censor individuals who leave employment for early retirement within the statutory regime, the significant negative effect of participation in the TC scheme on the survival rate in employment is very much reduced. Overall, the existing scheme does not pass the cost-benefit test.

The paper is structured as follows. We start with a literature review. In Section 3 we describe the institutional context, while in Section 4 we describe the sampling scheme and the data we used. Section 5 outlines the empirical strategy (identification and estimation). In Section 6 we present our empirical findings and in Section 7 the cost-benefit analysis. The final section summarizes the results and concludes.

\section{Literature Review}

It has been argued that workers might stay longer in the labour force if they could gradually reduce their working time at the end of their career (e.g. Schmid, 1998). Firms may voluntarily grant working time reductions if they realize that doing so allows them to keep valuable (firm-specific) competences and transfer know-how to younger employees (Eurofound, 2001; Kantarcl and van Soest, 2008). However, institutional constraints, such as provisions that the pension allowances depend on the last wage, or that a pension cannot be drawn upon while working part-time, may discourage older workers to reduce working time. In addition, employers may not be willing to award gradual retirement options (Hurd, 1996; Charles and Decicca, 2007; Gielen, 2009) so that government coercion or incentives may be necessary.

It is, however, far from guaranteed that eliminating institutional barriers and offering an explicit option to gradually retire by part-time work would keep older workers longer in the workforce or increase the

\footnotetext{
${ }^{3}$ In particular, Frölich (2007) demonstrates in a standard Kernel matching framework that endogenous sampling, contrary to choice-based sampling (i.e. sampling based on the treatment status), requires re-weighting the data both in the estimation of the propensity score and the treatment effect.
} 
total number of hours worked. First, pay of older workers may exceed their productivity. ${ }^{4}$ If employment protection is strong and early retirement schemes are not yet available for these workers, then firms may use subsidized part-time employment at the end of the career as an easy way to get gradually rid of these less productive workers. The gradual retirement scheme is then just a 'bridge' to (early) retirement and does not prolong the working career (Graf et al., 2011). This process may be reinforced if a phased reduction in working hours (i) does not lead to a proportional reduction in the workload (Devisscher and Sanders, 2007; Rudolf, 2014), (ii) signals a preference for early retirement (Machado and Portela, 2012), or (iii) just decreases labour market attachment. Finally, even if the withdrawal from the labour force is delayed, the total number of hours worked may still decrease. An hours-constrained worker who is not offered the possibility to reduce working time has two options: Either stop working altogether or stay working full-time. If such a worker is then offered the possibility to limit the number of hours worked, she increases the number of hours worked if the first option was chosen, but the reverse holds for the second option. The net effect on the number of hours worked depends on the relative size of these effects (Gielen, 2009; Graf et al., 2011).

The empirical literature studying the effectiveness of gradual retirement schemes based on counterfactual evaluations is very sparse. Graf et al. (2011) study the Austrian old age part-time (OAPT) scheme based on the conditional independence assumption (CIA) using propensity score methods (see e.g. Imbens and Wooldridge, 2009). They contrast all 6,142 men and 3,210 women who entered the OAPT scheme between 2000 and 2003 to a control group of 23,810 men and 28,651 women who were employed at least one day in 2000/2001 and to whom a hypothetical start date was assigned according to the simulation procedure described in Lechner and Wunsch (2008). ${ }^{5}$ They find that the OPAT scheme increases the number of days employed by 30 days on average during each of the first two years after entrance. However, in the fourth and fifth year the OPAT decreases the number of days employed by about 35 for women and by nearly 50 for men. Consequently, the cumulative effect over five years was negative. Moreover, these figures do not take into account that OAPT participants work part-time. In full-time equivalents the time worked diminished over five years by 26 (23) percentage points for men (women). On the other hand, the time spent in unemployment fell over this period by 37 (43)

\footnotetext{
${ }^{4}$ There is some evidence that declining productivity with age or deferred compensation schemes induce a pay-productivity gap for older workers. Hellerstein et al. (1999) did not find evidence for a pay productivity gap in the U.S., but a recent replication of this seminal analysis reports that pay exceeds productivity for workers older than 55 in the manufacturing sector (Hellerstein and Neumark, 2007). Aubert and Crépon $(2003,2006)$ establish similar results for this age group in France, Ilmakunnas and Maliranta (2005) for Finland, and Cataldi et al. (2012) and Vandenberghe et al. (2013) for Belgium. By contrast, no pay-productivity gap is found in Portugal and in the Netherlands (Cardoso et al., 2011; van Ours and Stoeldraijer, 2011). Frimmel et al. (2015) report evidence that in Austria employees of firms with higher labour costs for older workers leave the labour force at a younger age.

${ }^{5}$ As mentioned in the Introduction, this procedure has been criticised by Fredriksson and Johansson (2008).
} 
days for men (women). This is most likely because in the "block-model" these workers were not required to be made redundant, since they were not working anyway.

Huber et al. (2015) use the CIA to study the effect of introducing partial retirement in Germany. A novelty in their approach is that they consider the intention-to-treat effects of introducing the option of partial retirement at the firm level on the labour market outcomes of the employees in these firms. More specifically, they contrast the labour market outcomes between 2003 and 2009 of employees in firms that started offering partial retirement between 2000 and 2002 to firms that did not offer this opportunity in this period. To avoid that the composition of the workforce in these firms would be influenced by the introduction of the partial retirement scheme, the estimation was restricted to workers who had at least three years of tenure in these firms in June 2000. The authors report very different findings for West- than for East-Germany and attribute this difference to the difficult labour market conditions in the latter region. In West-Germany the option of partial retirement did not have any significant impact on the timing at which the labour market was left, neither did it induce any spillovers to younger employees. However, as in Austria, the "block-model" affected the pathway to retirement. Rather than transiting to unemployment prior to retirement participants entered the nonemployment block of the scheme. By contrast, in East-Germany older workers remained significantly longer attached to the labour market, which resulted in net savings for the public authorities through lower benefit expenditures and higher tax incomes and social security contributions. Moreover, presumably by the financial incentive to replace the partial retiree by unemployed workers, the scheme generated positive spill-overs by increasing employment stability and by lowering the likelihood of unemployment of younger women (but not of men).

Berg et al. (2015) also evaluate the German partial retirement scheme based on a difference-indifferences strategy which contrasts a younger control group of 50-54 year olds to a treatment group of 55-65 year olds in the pre-treatment period (1993-1998) and two post-treatment periods: 19992001 and 2002-2004, respectively a period in which the block-model was less and more intensively used. This analysis confirms that the block-model reduced the positive impact of the partial retirement scheme on the probability of staying in the labour force. During the period of less intensive use of the block-model male participants remained 1.8 years longer employed than non-participants, while this effect fell to 1.2 years in the period of more intensive use (if the time not working during the second phase of the block model is not considered as employment). The employment of female participants was, however, not affected in the former period, while it even declined by 0.2 years in the latter. The findings of Huber et al. (2015) refer to the period of more intensive use of the block-model. Nevertheless, it is not easy to compare the two studies, since, in contrast to Huber et al. (2015), Berg 
et al. (2015) did not stratify the analysis between West- and East-Germany and did not consider spillovers on the employment of younger individuals.

Finally, Elsayed et al. (2015) use vignettes in a web-based survey of 3,611 Dutch public servants to evaluate the introduction of an early retirement scheme in the Netherlands. ${ }^{6}$ In this survey respondents get different vignettes of hypothetical retirement scenarios, including gradual retirement. For each of these vignettes the respondents should state at which age they would retire. In the vignettes that contain the gradual retirement scenario working time is reduced by $50 \%$ and workers are entitled to half of the full-time pension. The level of the pension replacement rate and its accrual with age varies according to the considered scenarios. As to provide causal interpretations of the systematic relations between the responses and the scenarios, different scenarios were randomly assigned to individuals. The study finds that a gradual retirement scheme would induce workers to retire on average one year later. However, total lifetime labour supply would still fall by 3.4 months, because the reduced working hours within the gradual retirement scheme dominate the delayed exit from the labour force.

\section{Gradual Retirement in Belgium: Time Credit Beyond the Age of 50}

In 2002 the Career Break scheme, in place since 1985 and available in both the private and public sector, was reformed and relabelled "Time Credit" (TC) scheme in the private sector. The schemes aim at generating a better work-life balance by enabling and encouraging employees, even without needing to specify a specific motive, to slow down their working pace. Workers younger than 50 can temporarily reduce their working time, while older workers can take advantage of these schemes without any time limit, as to enable a more gradual transition to retirement (Devisscher, 2004). We focus on a description of the main features of the latter section of the TC scheme, also called the endof-career TC. We restrict our description to the regulations in place during the 2002-2007 period. This covers the relevant period for the empirical analysis in this research. In 2015, the scheme is still in place, but most of the eligibility conditions have been strengthened considerably. Most notably, the age of eligibility was raised to 55 years in 2012 and starting from 2015 this age will gradually increase to 60 in 2019. Nevertheless, eligibility at 55 remains possible under certain circumstances. ${ }^{7}$

Individuals older than 50 who are employed in a private sector firm are under certain conditions entitled to reduce their working time to $80 \%$ or $50 \%$ of a full-time, or even completely. They are entitled to a lump-sum state subsidy that partially compensates for the earnings loss that the transition

\footnotetext{
${ }^{6}$ The initial sample consisted of 13,151 e-mail addresses. Hence, the response rate was only $27 \%$.

${ }^{7}$ This is the case for workers in arduous professions, in case of partial work incapacity, in restructuring firms, or in cases that the employer organisations and trade unions have concluded a Collective Agreement and the employers agree to pay the subsidy to which participants are entitled that would otherwise be due by the Social Security scheme.
} 
to part-time work involves. Notice that the possibility to completely stop working was much less used than the other regimes, because the subsidy was only $34 \%$ higher than the one obtained for the $50 \%$ regime and more generous early retirement schemes were available in this case, although generally only at older ages (see below). Since our interest is in gradual retirement, we do not further consider this regime. The main eligibility conditions for the end of career TC scheme were the following:

1. Being at least 50 years old at the start of the working time reduction;

2. Being full-time employed during the year prior to entry for the $20 \%$ regime; being employed for at least $75 \%$ of a full-time schedule for the $50 \%$ regime;

3. At least 5 years of tenure in the same firm;

4. At least 20 years of labour market experience;

5. Consent of the employer, if the number of employees in the firm is at most 10 or, in case the firm employs 11 workers or more, if the fraction of employees in the TC is strictly larger than $5 \%$ (can be revised by a collective agreement);

6. Notification to the employer at least 3 months prior to the working time reduction.

If these eligibility conditions are satisfied the employee is entitled to a monthly lump-sum subsidy of, (i) in case of the $20 \%$ regime, $€ 224^{8}$ for singles with or without dependent children and $€ 186$ for other household types, and, (ii) in case of the $50 \%$ regime, $€ 400$ for all household types. For the sample of TC beneficiaries analysed in this research, this results in a median replacement rate of $83 \%$ of the fulltime gross wage for those working $80 \%$, and of $57 \%$ for those working half-time. As a comparison, the replacement rate after the top-up was $70 \%$ for a half-time worker in Germany and $75 \%$ when working $40 \%$ to $60 \%$ in Austria. The replacement rate is therefore lower for a median worker in Belgium, but, since the subsidy is lump-sum in Belgium and proportional in Germany and Austria, it is higher for lowwage workers in Belgium. ${ }^{9}$

The end of career TC was very popular. The number of participants grew steadily from 8,700 in 2002, the year it was introduced, to 88,000 in 2011 , the year prior to the one in which the age of eligibility was raised to 55. To compare, in 2001, the year prior to the reform, the number of private sector beneficiaries older than 50 in the Career Break scheme amounted to $18,745 .{ }^{10}$ As a share of private sector employees aged 50 or more, TC participation steadily increased from $2.5 \%$ in 2002 to $16.0 \%$ in 2011. This growth is related to both, the rising employment rate of older women in this period and to the increased generosity of the TC relative to the Career Break. The subsidy amount was raised by

\footnotetext{
${ }^{8}$ All $€$ in the text are indexed by the CPI and expressed in constant 2004 euros.

${ }^{9}$ For the $20 \%(50 \%)$ regime the first and third quartile of this replacement rate ranges between $81 \%(55 \%)$ and $91 \%(62 \%)$, while the $90^{\text {th }}$ percentile of this replacement rate, i.e. for low-wage workers, is as high as $96 \%$ (75\%).

${ }^{10}$ Only new beneficiaries entered TC. The stock of existing beneficiaries remained entitled to the Career Break.
} 
about $20 \%$ in the $50 \%$ regime and by nearly $40 \%$ in the $20 \%$ regime. The share of female TC participants steadily grew from $27 \%$ in 2002 to $52 \%$ in $2011 .{ }^{11}$

Apart from the lump-sum subsidy, another major benefit for TC participants is that for the determination of the level of replacement income in the branches of Social Security, such as (early) statutory retirement and unemployment, TC beneficiaries are assimilated to workers with the same time schedule as before the working time reduction. This means that relative to their current income, TC participants earn a much higher replacement income (i.e. the ratio of the full-time (early) pension to the part-time labour earnings plus the TC allowance) if they leave employment for (early) retirement than workers who remain full-time employed. As a consequence, the incentive to stop working is significantly enhanced, especially for workers who choose the $50 \%$ TC regime: the numerator of their replacement income is unaffected, while the denominator significantly decreases. In line with these incentives, we will show in the empirical analysis that the participation in TC eventually enhances the transition to early retirement. Moreover, because these incentives were different across the different early retirement schemes, they also altered the pathway to early retirement. To understand this point, we briefly describe the different early retirement options in Belgium and the impact of the TC on the benefit level in these options.

In Belgium there are essentially three early retirement schemes in the private sector: early retirement within the statutory regime, the conventional pre-retirement scheme (also known as the "bridge pension") and the, so-called, "Canada Dry" system. ${ }^{12}$ In the period of analysis, early retirement within the statutory regime started from age 60 after minimum 35 years of employment experience. ${ }^{13}$ This is an early retirement scheme, because the statutory retirement is normally entered after 45 years of labour market experience or at age 65 for men. Women retired between 63 and $65 .{ }^{14}$ The benefit level is determined as at the statutory age and provides in case of a career of 45 years a replacement rate of $75 \%$ or $60 \%$, depending on whether the partner of the beneficiary, if any, has any (replacement) income or not. This amount is proportionally reduced if the career is shorter than 45 years and is bracketed by a floor and a ceiling amount. Due to relatively low generosity of this scheme for full-time workers in the private sector, ${ }^{15}$ take-up in the two alternative regimes, especially the bridge pension, is much more important. For workers having more than 20 years of employment experience, the bridge pension is available from age 60 for all workers and from 58 in case of a sectoral collective agreement,

\footnotetext{
${ }^{11}$ Sources: year reports of the RVA/ONEM 2001-2011.

12 "Canada Dry" refers to publicity for the drink Canada Dry: "It has the colour of Whisky, but it is not Whisky".

${ }^{13}$ This experience requirement was gradually increased from 20 to 34 years by steps of two years in each calendar year between 1997 and 2004, and, eventually, by one year to 35 years in 2005.

${ }^{14}$ The statutory retirement age for women was raised by one year every three years from 60 before 1997 to 65 from 2009 onwards.

${ }^{15}$ For public sector employees the scheme is much more generous and, hence, more widely used.
} 
mostly concluded in the industrial committees ${ }^{16}$ of the manufacturing sector. In restructuring firms and for arduous professions, the age condition could drop to 50, 52 or 55, depending on the sectoral agreement. Because of a supplement equal to half of the difference between the unemployment benefit (UB) and the wage, the bridge pension is more attractive, at least if they are not in TC (see below). The "Canada Dry" is an unofficial early retirement scheme in which the employer pays, as in the bridge pension, a supplement to the UB. This scheme is more flexible for the employer, because it does not impose an age limit among others (Albanese and Cockx, 2015). Since there is no obligation for the worker to report the supplement to the UB she obtains, no official figures on the use of the Canada Dry scheme are available.

As already mentioned, the UB and the statutory (early) retirement pension for a beneficiary of TC is calculated on the basis of the fictitious earnings that the employee would have had if she would not have reduced her working time. By contrast, TC does decrease the benefit level of the bridge pension. Even if the entitlement to UB is based on the fictitious earnings, the supplement is equal to $50 \%$ of the difference between the effective part-time wage in TC and the UB and, hence, much lower or even zero if the UB is higher than the wage, which can happen in the $50 \%$ TC regime. ${ }^{17}$ Consequently, to the extent that the more restrictive age and experience requirements are satisfied, the statutory early retirement scheme is relatively more attractive than the bridge pension (and the Canada Dry) for beneficiaries of TC than for full-time workers. Moreover, the statutory early retirement can be entered without consent of the employer, while the bridge pension, Canada Dry or plain unemployment does require this consent, because the employer must then also compensate for the dismissal, which is costly for these older workers with substantial seniority. This explains why we find in the empirical analysis that TC increases the likelihood of ending the career through the statutory early retirement, especially in the $50 \%$ TC regime.

\section{Data \& Sample Selection}

\subsection{Database}

We use rich individual data that were obtained by merging administrative registers of the diverse Social Security institutions and of the National Register containing all Belgian inhabitants. The database became more comprehensive over time. From as early as 1957 until 1998 we have for employees in

\footnotetext{
${ }^{16}$ Industrial committees are organized for each type of worker at the sectoral level. In these committees trade unions and employer organizations negotiate the collective agreements. These agreements are binding for all workers belonging to this industrial committee, irrespectively of whether they are unionized and, hence, represented in the negotiation.

${ }^{17}$ For instance, heads of household and singles with more than 20 years of experience and aged 55 or more are entitled to UB with a replacement rate of at least $60 \%$ (slightly higher for heads) and of $55 \%$ for cohabitants of heads aged 58 or more (with floor and cap). For younger workers or worker with less experience the level of UB is somewhat lower.
} 
the private sector yearly information on earnings, the number of working days and hours (in case of part-time work) and the worker type (blue or white collar). From 1998 onwards this information is available on a quarterly basis (measurement at the end of each quarter), not only for employees in the private sector, but also in the public sector. In addition, from then onwards it also contains on limited firm information (size and sector), the industrial committee to which the worker belongs to (see footnote 16), the timing of self-employment spells, on UB receipt, as well as on participation in the Career Break, TC schemes and early retirement schemes. Finally, since 2003 the data have been complemented by information on sick leave, on receipt of statutory (possibly early) retirement benefits and replacement income in case of disability, occupational diseases or accidents. Finally, since 1998 the National Register provides yearly information on December 31 on individual and household characteristics, such as age, gender, nationality, district of residence, household size (by age group) and type (single or couple, with or without children). The observation period in this study ends in the last quarter of 2011.

\subsection{Sample Selection}

In this study we base our analysis on a sample that was drawn with the purpose to evaluate the effect of a wage cost subsidy for employees in the private sector aged 58 years or more. The Belgian government introduced this subsidy in 2002 to enhance the employment of older workers (Albanese and Cockx, 2015). To that end a representative sample was drawn of 243,655 individuals born between the 1st of April 1941 and the 31st of March 1950, i.e. aged between 52 and 61 in 2002. Because in Belgium many individuals are already inactive in that age bracket, the sample was not only stratified according to gender, but also into 9 birth cohorts $c(=1,2, \ldots, 9)$ and 5 strata $r(=1,2, \ldots, 5)$. These strata were defined according to employment status in the private sector and the earned wage in the period around the 2002 reform. This stratification aimed at over-representing groups that are relatively rare in that age bracket and more responsive to the labour market policy reform: low-wage employees in the private sector and individuals transiting in and out of employment during this period. In Appendix B more details on this stratification can be found. Because the stratification involves outcome variables of interest, it is endogenous and it is well known that consistent estimation then requires to appropriately weigh the data in these strata (e.g. Manski and Lerman, 1977; Cameron and Trivedi, 2005). If we denote the sampling weight for individual observation $i$ belonging to birth cohort $c$ and to substratum $r$ by $W_{c r, i}$, then

$$
W_{c r, i}=\frac{N_{c r}}{N} * \frac{n}{n_{c r}}
$$


where $N_{c r}$ denotes the size of the population in substratum $c r,{ }^{18} n_{c r}$ the corresponding sample size, $N \equiv \sum_{c=1}^{9} \sum_{r=1}^{5} N_{c r}$ the total population size and $n$ the corresponding sample size. As to avoid cumbersome notation, gender is not explicitly referred to. The weighting formula comes from a double re-weighing, within and between cohorts. ${ }^{19}$

In this study we aim at evaluating the impact of participating in TC on the survival rate in employment. The TC scheme was introduced in 2002. Nevertheless, we start the evaluation only from 2003 and this for two reasons. First, we wish to consider the scheme at a moment when it is well established and the rules are well known. Second, we also aim at integrating the incidence on sick leave as a second outcome. As mentioned before, information on sick leave is only available since 2003.

We evaluate the impact of TC spells that started in 2003 and 2004. In principle we could also consider TC that started in later years. However, the available sample participation in TC declines over time and these additional treatments would not be helpful in identifying the long-run effects (up to eight years after the start of the treatment) in which we are particularly interested. ${ }^{20}$

Because the TC can start at any moment within the eight years of analysis, there is a dynamic assignment into treatment which induces, as mentioned in the Introduction, some intricate methodological issues (Fredriksson and Johansson, 2008). In particular, individuals who start the treatment later on may confound the control group. Besides, participants may anticipate the treatment. In Section 5 we will explain in more detail how we deal with these issues. At this point we limit the discussion to what this implies for the definition of treatment and control groups.

First, as to model this dynamic assignment process and to take it into account we must split up the assignment period into sub-periods. If the sample size would not be an issue, one would split up this period as finely as possible. However, within the available sample the transition rate into TC is relatively low, so that we ran into inference problems when we defined quarterly intervals. We therefore chose to consider yearly intervals. Furthermore, to have a sufficiently high number of treated individuals and increase precision we perform two separate analyses on two treatment groups, depending on whether the TC started in 2003 or 2004 , and pool the estimates.

A second important issue is anticipation. Participation in TC is always anticipated, because the employer must be notified at least three months before the start. This means that the treatment

\footnotetext{
${ }^{18}$ We have information on the population sizes in each substratum, i.e. on $N_{c r}$.

19 First, to restore the representativeness within the cohorts we reweigh the units within each cohort by $W_{c r}^{c}=\frac{N_{c r}}{N_{c}} * \frac{n_{c}}{n_{c r}}$ (where $N_{c}$ and $n_{c}$ is the size of the cohort in the population and in the sample). To make the cohorts in the sample representative for the population, we weigh each cohort a second time: $S W_{c r}=W_{c r}^{c} * \frac{N_{c}}{N} * \frac{n}{n_{c}}$, so that $W_{c r}=\frac{N_{c r}}{N} * \frac{n}{n_{c r}}$.

${ }^{20}$ The decline in the number of participants is due to the ageing of our sample, exiting the labour force over-time. Official statistics from RVA/ONEM show that until 2005 the share of employees in TC steadily increased by about 2 pp in each year.
} 
actually starts before the working time reduction related to the TC scheme is implemented. To take this into account we therefore assume that a treatment actually starts one quarter earlier than it contractually does. Hence, with this new assumption, the period that determines treatment is advanced one quarter, so that an individual is defined to be member of the first treatment group if she actually participates in a TC at the end of the third quarter 2003 (2003Q3) while she did not at the end of 2002Q3. However, shifting the start of the treatment is not sufficient to deal with anticipation, because without information on when the treatment actually started, it is impossible to determine whether it had any impact on the outcome between the actual and contractual start of the treatment i.e. exit from employment. ${ }^{21}$ We therefore impose the additional assumption that individuals never leave employment in this period. This assumption biases the treatment effect upwards (i.e. a higher survival rate in employment for the treated). However, this bias is arguably small, because it is unlikely that individuals who agreed with the employer that they would start TC within the next quarter would eventually decide to stop working beforehand. This would presumably only occur for reasons of force majeure, such as an accident.

In order to enhance the comparability of treated and control groups, we impose that members of both groups should satisfy criteria slightly stricter than those that determine eligibility to TC:

1. Being employed in a firm with at least 20 employees at the end of 2002 (2003);

2. Have at least 5 years of tenure in the same firm at the end of 2002 (2003);

3. Have at least 20 years of private sector labour market experience at the end of 2002 (2003);

4. Being full-time employed in all four quarters of 2002 (2003);

5. Being employed in the private sector at the end of 2003Q3 (2004Q3);

6. Not being on sick leave at the end of 2003Q3 (2004Q3).

The fifth selection criterion is imposed at the end of each assignment period into treatment, because it is automatically satisfied for the members of the treatment groups, so that it is natural to impose it on the members of the control groups as well. The other criteria are slightly more restrictive than the TC eligibility conditions (Section 3), so that a few treated individuals are eliminated from our initial selection. We are slightly more restrictive for the following reasons: (i) Eligibility conditions 1-4 are uniformly imposed at the end of the year preceding the contractual start of the TC so that the same conditions apply to all treated and control units; (ii) Because the data contain only basic information about firm characteristics, we aim at restricting the analysis to sufficiently large firms in which the use of TC does not require the consent of the employer (Section 3). If no consent is required, it is less likely

\footnotetext{
${ }^{21}$ Any form of employment is considered: part-time or full-time, public, private or self-employment, but between the actual and contractual start of TC the worker must be full-time employed, since otherwise she would not be eligible to TC at the contractual start of TC.
} 
that the use of TC is selective in firm characteristics, which therefore enhances the internal validity of the evaluation. However, according to the rules, no consent is required in firms with more than 10 employees. Nevertheless, we include only firms that employ at least 20 workers. This is because the available data on firm size are grouped in intervals that do not allow identifying firms with strictly more than 10 employees; (iii) While regulations do not impose that the labour market experience should be accumulated in the private sector, we do because we do not have information on early experience outside the private sector; (iv) We impose full-time employment in the last year prior to contractual assignment into treatment, while for the $50 \%$ TC regime the requirement is only to have worked at least $75 \%$ of a full-time job. This is done to consider only individuals who are eligible to both regimes; (v) Finally, we impose the last condition on sickness in 2003 Q3 (2004Q3) because we want to contrast the impact of the benchmark outcome, i.e. survival in employment, to a more restrictive variant that considers survival in employment without being on sick leave. If we would not impose this, some of the selected individuals would not be in the risk set of this second outcome at the start of the evaluation period. Imposing this condition only very marginally affects the sample selection. Finally, note that we do not impose the age condition, because our sample only contains individuader than 50.

Based on these selection rules we retain, for men, 1,227 treated and 29,791 control units. For women the sample size is smaller: 762 treated and 9,658 control units. By using the sampling weights defined in Equation (1) we find that this represents in the Belgian population about 5,124 treated and 90,387 control units. Note that the control units in the two years of analysis partly consist of same individuals, while treated units are always different.

\subsection{Descriptive Analysis}

As described in the previous Section, our sample is composed of individuals satisfying the eligibility conditions to the TC one year prior to the selection of 2003 (or 2004). Consequently, the individuals considered in the analysis are relatively homogeneous in the dimensions that matter for these conditions such as employment experience in the private sector (20 years), tenure (5 years), firm size (> 20 employees) and full-time work in the year prior to the selection (2002 or 2003). Even if the individuals in the two groups are already homogenous in several dimensions, they still differ significantly in a number of other dimensions. This justifies the use of the Inverse Probability Weighting (IPW) estimator proposed in the next section. The full list of variables on which we condition in the analysis are shown in Table 1. We distinguish between men and women, because the analysis is performed separately for these groups. The units in the treatment and control groups for the 2003 and 
2004 analyses are pooled. Note that $54.3 \%$ of the treated are in the $50 \%$ TC regime, the remainder in the $20 \%$ TC regime.

Table 1: Descriptive Statistics of Selected Treated and Control Groups (weighted by $W_{c r, i}$ )

\begin{tabular}{|c|c|c|c|c|c|c|c|}
\hline & & & & & \multirow{2}{*}{\multicolumn{3}{|c|}{ Women }} \\
\hline & \multicolumn{3}{|c|}{ Men } & & & \\
\hline & & TREATED & CONTROL & $\begin{array}{l}\text { P-value: } \\
\text { equality }\end{array}$ & TREATED & CONTROL & $\begin{array}{l}\text { P-value } \\
\text { equality }\end{array}$ \\
\hline \multirow{20}{*}{$\begin{array}{l}\text { Status one } \\
\text { year before } \\
\text { selection } \\
(2002 \text { or } \\
2003)\end{array}$} & Firm size: $20-99$ & $18.8 \%$ & $30.2 \%$ & 0.000 & $26.4 \%$ & $32.9 \%$ & 0.000 \\
\hline & Firm size: 100-999 & $30.9 \%$ & $37.8 \%$ & 0.000 & $27.9 \%$ & $36.7 \%$ & 0.000 \\
\hline & Firm size: > 1000 & $50.4 \%$ & $32.0 \%$ & 0.000 & $45.8 \%$ & $30.4 \%$ & 0.000 \\
\hline & Household: Other & $11.2 \%$ & $13.4 \%$ & 0.043 & $35.7 \%$ & $40.8 \%$ & 0.009 \\
\hline & $\begin{array}{c}\text { Household: } \\
\text { Couple with children } \\
\end{array}$ & $46.4 \%$ & $48.1 \%$ & 0.326 & $24.3 \%$ & $24.4 \%$ & 0.984 \\
\hline & $\begin{array}{c}\text { Household: } \\
\text { Couple without children }\end{array}$ & $42.4 \%$ & $38.5 \%$ & 0.025 & $39.9 \%$ & $34.9 \%$ & 0.010 \\
\hline & Age & 55.5 & 55.8 & 0.000 & 55.4 & 55.7 & 0.000 \\
\hline & Blue collar & $23.5 \%$ & $30.5 \%$ & 0.000 & $10.3 \%$ & $12.4 \%$ & 0.095 \\
\hline & Av. Full-Time Hourly wage & $€ 20.7$ & $€ 22.5$ & 0.000 & $€ 17.5$ & $€ 17.7$ & 0.365 \\
\hline & Belgian & $98.0 \%$ & $95.0 \%$ & 0.000 & $97.2 \%$ & $96.3 \%$ & 0.236 \\
\hline & Household size & 2.6 & 2.7 & 0.022 & 2.1 & 2.1 & 0.630 \\
\hline & Region: Brussels & $5.7 \%$ & $7.1 \%$ & 0.079 & $15.4 \%$ & $19.2 \%$ & 0.009 \\
\hline & Region: Flanders & $70.2 \%$ & $64.8 \%$ & 0.001 & $51.9 \%$ & $48.0 \%$ & 0.055 \\
\hline & Region: Wallonia & $24.1 \%$ & $28.2 \%$ & 0.007 & $32.8 \%$ & $32.8 \%$ & 0.968 \\
\hline & Sector: Trade, transport, hotel & $15.0 \%$ & $20.7 \%$ & 0.000 & $21.6 \%$ & $24.6 \%$ & 0.073 \\
\hline & Sector: Bank, business services & $44.6 \%$ & $18.7 \%$ & 0.000 & $39.7 \%$ & $24.5 \%$ & 0.000 \\
\hline & Sector: Other services & $4.0 \%$ & $6.8 \%$ & 0.000 & $21.5 \%$ & $26.3 \%$ & 0.004 \\
\hline & $\begin{array}{l}\text { Sector: Manufacturing, } \\
\text { Agriculture, Construction }\end{array}$ & $31.8 \%$ & $47.5 \%$ & 0.000 & $17.2 \%$ & $24.6 \%$ & 0.000 \\
\hline & Sector: Construction (for men) & $4.6 \%$ & $6.3 \%$ & 0.018 & - & - & - \\
\hline & $\begin{array}{l}\text { Early retirement propensity } \\
\text { in the Industrial Committee* }\end{array}$ & $-1.0 \%$ & $1.8 \%$ & 0.000 & $-3.6 \%$ & $-2.0 \%$ & 0.000 \\
\hline $\begin{array}{c}5 \text { years before } \\
\text { selection }\end{array}$ & Av. Full-Time Hourly wage & $€ 20.2$ & $€ 22.3$ & 0.000 & $€ 17.0$ & $€ 17.3$ & 0.249 \\
\hline $\begin{array}{c}13 \text { years } \\
\text { before } \\
\text { selection }\end{array}$ & Years with the same employers & 10.6 & 11.8 & 0.000 & 11.1 & 11.7 & 0.000 \\
\hline $1990-1997$ & Av. Working time (\%) & 98.0 & 97.5 & 0.002 & 95.8 & 96.2 & 0.272 \\
\hline \multirow{2}{*}{ 1957-1997 } & Experience in years & 31.2 & 31.0 & 0.071 & 30.1 & 29.7 & 0.023 \\
\hline & Av. Earnings in the year & $€ 29,010$ & $€ 30,247$ & 0.000 & $€ 23,831$ & $€ 23,368$ & 0.141 \\
\hline \multirow[b]{2}{*}{$\mathrm{N}$ individuals } & Sample Size (Unweighted) & 1,227 & 29,791 & & 762 & 9,658 & \\
\hline & $\begin{array}{l}\text { Represented Population Size } \\
\text { (Weighing Sample Size - } W_{c r, i} \text { ) }\end{array}$ & 3,863 & 75,778 & & 1,261 & 14,609 & \\
\hline
\end{tabular}

* Estimation based on a linear probability model using the complete sample of 243,655 individuals (cf. Section 4.2) on the period 1998q1-2002q3. We regress "transiting to a bridge pension" on dummies for the Industrial Committee (IC) or Nace if IC is missing, birth cohort dummies and gender. The retained variable contains the coefficients (i.e. marginal effect) of the IC dummies. In a sensitivity analysis we replace these marginal effects (estimated by OLS) with the predicted probabilities (estimated by a probit model) at the average Xs. Results are very similar and available upon request.

First, we consider the variables measured the last quarter prior to the start of the selection (i.e. $2002 Q 3$ and 2003Q3 for the first and second analysis). Treated units tend to work in larger firms, live with a partner without dependent children, are slightly younger, earn a lower hourly wage and, are more likely to be Belgians living in Flanders. Finally, they are concentrated in specific economic sector such as the banking and business related services and more in general in white-collar work. The industrial committees where they tend to work are the ones where bridge pension schemes are less common. 
Second, we consider both the recent and less recent employment history. ${ }^{22}$ In line with the year before the selection, treated individuals earn a lower hourly wage five years before selection and had lower average annual earnings since 1957 (the difference is statistically significant only for men). Tenure in the same firm is slightly shorter and total accumulated working experience since 1957 is slightly more important. If we consider also the 5 years of tenure prior to the sample selection, then the individuals in the sample have on average about 36 years of experience, which makes many of them already eligible to the statutory early retirement. ${ }^{23}$

Figure 1: Raw Sample - Differences in Survival Rate in Employment by Treatment Status

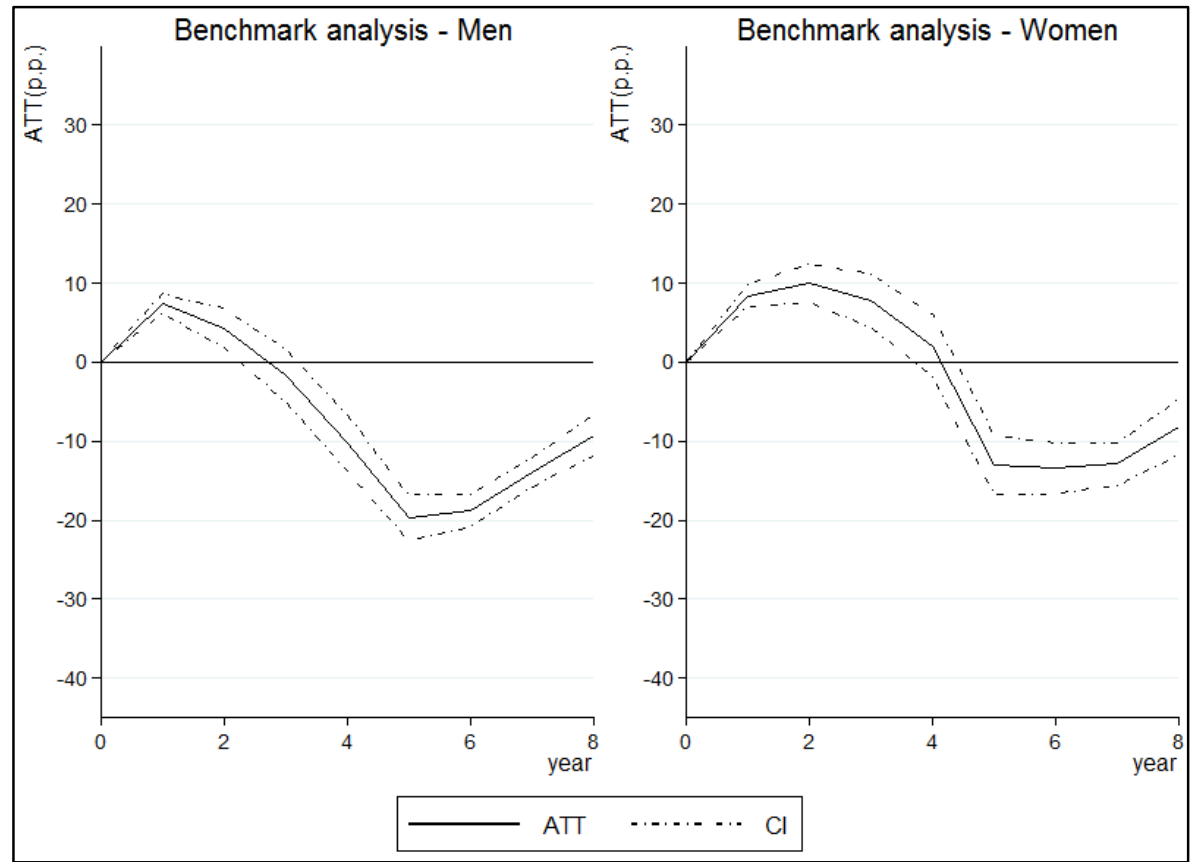

Differences in survival rate by treatment status (ATT). The survival is defined with respect to employment and the point estimates are expressed in percentage points (pp). Pooled estimates for the treated samples of 2003 and 2004. The pooled sample is composed of 1,227 male (762 female) treated and 29,791 male (9,658 female) control units. Standard errors are cluster robust to take into account correlation between the same individuals in the two sample.

To have a first idea on the possible effect of the TC we compare in Figure 1 the survival rate in employment of treated and control individuals following the treatment. Treated individuals show a higher likelihood to survive in employment in the first two years (four for women) but the cumulated effect on the survival rate reverses and reaches a maximum of $-20 \%$ for men and $-15 \%$ for women in the sixth/seventh year. From this descriptive evidence it seems that individuals entering the schemes tend to remain in employment for a few years longer. This is consistent with the hypothesis that they initially continue working as to accumulate pension rights of a full-time worker. However, as time

\footnotetext{
${ }^{22}$ The variables referred to the employment history are not combined to keep information coming from different sources and periods with missing information separate. For example, information from 1998 comes from payroll-tax-administration (quarterly data), while from 1957 to 1997 from pension registrations (yearly data).

${ }^{23}$ Note, however, that the measure of experience in Table 1 is likely overestimating the definition used in the determination of the eligibility to a statutory pension, and thus overstates the likelihood of eligibility.
} 
evolves they are more likely to become eligible to the statutory early retirement scheme and, because they earn a higher replacement income than those who did not take-up TC (Section 3) they eventually withdraw faster from the labour market.

By the end of the period of analysis, i.e. 2011q3, 93.7\% of the treated units are no longer employed while this share is only $81.8 \%$ for the control sample. The specific exit destinations for the treated and control group can be found in Table 2. The main difference in behaviour is that control units tend to use the bridge pension as the most common pathway to retirement while for the treated units this is the statutory early retirement. This is related to how participation in TC affects the benefit entitlement within these different early retirement schemes. As explained in Section 3, the statutory early retirement pension for a beneficiary of TC is calculated on the basis of the fictitious earnings that the employee would have had if she had not reduced her working time. By contrast, TC does decrease the benefit level of the bridge pension. In Section 6 we will show that the descriptive evidence in this section is qualitatively not much affected if we take the compositional differences between treated and control groups into account.

Table 2: Exit Destinations (\%) by Treatment Status

\begin{tabular}{|c|c|c|}
\cline { 2 - 3 } \multicolumn{1}{c|}{} & Treated (\%) & Controls (\%) \\
\hline Statutory early retirement & 48.5 & 23.2 \\
\hline Bridge pension & 34.2 & 38.5 \\
\hline Unemployment & 1.7 & 2.0 \\
\hline Statutory retirement at the legal retirement age & 4.1 & 8.8 \\
\hline Other inactivity & 5.2 & 9.3 \\
\hline Never exit & 6.3 & 18.2 \\
\hline Sample size (Unweighted) & 1,989 & 39,449 \\
\hline
\end{tabular}

Exit destinations of the treated and control group in the quarter after leaving employment in \% of total exits. Note that some individuals never exit and remain employed until the end of the period of analysis. Unemployment contains the Canada Dry. Other inactivity includes among others people who die and a small minority $(0.6 \%)$ of exits to disability schemes. Differently from other countries, these schemes are not particularly generous compared to the early retirement scheme.

\section{Empirical Strategy}

\subsection{Notation and the Treatment Effect of Interest}

We are interested in estimating the average treatment effect on the treated (ATT) in TC on the survival rate in employment. ${ }^{24}$ An exit from employment is defined as soon as an individual is not observed in employment at the end of a quarter. In case of an exit, $93 \%$ of the individuals never return to employment before the statutory retirement age. This means that this exit is, in most cases, equivalent to an early withdrawal from the labour market, i.e. a pathway to retirement. We impose that once a

\footnotetext{
${ }^{24}$ As a second outcome the survival rate in employment without sickness leave is considered. Since this does not affect the empirical strategy we ignore this in the further discussion of this section.
} 
treatment has started it cannot be reversed. This means that the treatment status is not affected in the rare cases that the TC scheme is left immediately after having entered it.

This evaluation problem is very similar to the one described by Vikström (2014). Since we follow Vikström's methodology closely, the exposition in this section is also analogous. The essential differences are the following. First, we generalize his procedure to allow for the endogenous sampling present in our data. Second, we do not base the evaluation on a flow sample of individuals in a state, but instead a stock sample of individuals who have been employed for at least 5 years at sample selection. ${ }^{25}$ If we normalize time to zero at the beginning of the two periods of analysis (i.e. at the end of $2002 Q 3$ and $2003 Q 3$ and consider the residual duration from then, we argue that the analysis does not require any adjustment. Third, we propose a different trimming rule for the determination of a common support for treated and control units. Finally, we propose a slightly different bootstrap procedure for inference on the pooled sample.

Figure 2: Graphical Representation of the Notation

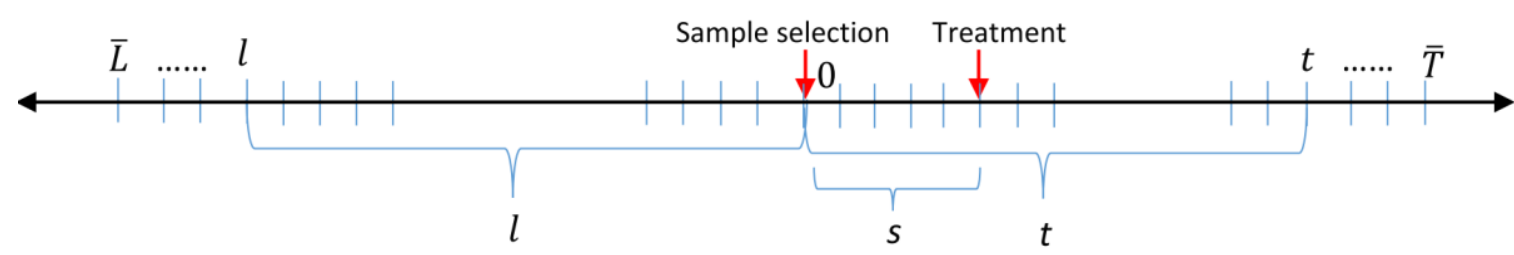

In Figure 2 we provide a graphical representation of the introduced notation. Let $t \in\{1,2, \ldots \bar{T}\}$ denote the number of years since sample selection (i.e. at the end of $2002 Q 3$ and $2003 Q 3$ ) and $l \in\{1,2, \ldots, \bar{L}\}$ the elapsed number of years in employment at this start. $\bar{T}$ and $\bar{L}$ are the maximum number of years in employment respectively, after and before selection. In the data $l \geq 5$, because this is an eligibility condition for the TC and a sample selection criterion (Section 4.2). The random time since sample selection until the start of the treatment, i.e. entry in TC, is denoted by $S$ and its realization by $s$, where $s \in\{0,1, \ldots, \bar{S}\}$ and $\bar{S} \leq \bar{T} . Y_{l+t}(s)$ is equal to one in case employment is left in year $l+t$ and treatment started in year $l+s$, and zero otherwise. $Y_{l+t}(\infty)$ denotes the potential outcome in year $l+t$ if never treated and $Y_{l+t}$ the observed outcome. $\bar{Y}_{l+t}(s) \equiv\left\{Y_{1}(s), Y_{2}(s), \ldots, Y_{l+t}(s)\right\}$ and $\bar{Y}_{l+t} \equiv$ $\left\{Y_{1}, Y_{2}, \ldots, Y_{l+t}\right\}$ denote, respectively, the sequence of potential and of observed outcomes.

\footnotetext{
${ }^{25}$ The 5 years of tenure condition is imposed at the end of 2002 (2003) for the first (second) treatment and control group, while sample selection requires the individual to be employed at the end of 2003Q3 (2004Q3) - the second condition mentioned in Section 4.2. Thus, at the end of 2003Q3 (2004Q3) 99.75\% of the selected individuals have at least 5.75 years of tenure and 20.75 year of labour market experience. We ignore for simplicity this detail in the exposition of the empirical strategy.
} 
We aim at identifying the average treatment effect on the treated (ATT) of treatment $s$ years after sample selection against the counterfactual of never being treated on the residual survival in employment until year $t>s$, given survival in employment until sample selection:

$$
\begin{gathered}
\forall t>s: A_{t}(s) \equiv E_{L}\left\{E\left[\bar{Y}_{L+t}(s)=0 \mid S=s, \bar{Y}_{L+s}(s)=0\right]\right. \\
\left.-E\left[\bar{Y}_{L+t}(\infty)=0 \mid S=s, \bar{Y}_{L+s}(s)=0\right] \mid L \geq 5\right\}
\end{gathered}
$$

This extends the ATT as parameter of interest to evaluation of a stock sample. Since in a stock sample individuals may have a different elapsed duration, the conditional expectation is taken over these elapsed durations, conditional on being employed for at least 5 years to take into account that one needs at least 5 years of tenure to be eligible for TC. Observe that $E\left[\bar{Y}_{L+t}()=0 \mid. S=., \bar{Y}_{L+s}()=0.\right]=$ $\operatorname{Pr}[T>L+t \mid S=., T>L+s]$ holds, i.e. the conditional probability of surviving $L+t$ years in employment given survival until $L+s$. In case $L=0$, Equation (2) reduces to the corresponding expression in Vikström (2014) for a flow sample. ${ }^{26}$

\subsection{Identification}

In order to identify $A_{T T}(s)$ we use two identifying assumptions: $\mathrm{ClA}$ and no anticipation (NAA). These assumptions can be formalized as follows:

$$
\boldsymbol{C I A} \forall l>5, \forall s, \forall t>s: S \perp Y_{l+t}(s) \mid X
$$

and

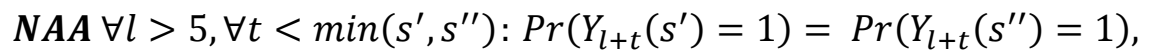

The latter condition means that individuals do not alter their behaviour in response to a future assignment to the treatment. Based on these assumptions Fredriksson and Johansson (2008), Crépon et al. (2009) and Vikström (2014) prove that for $l=0 A T T_{t}(s)$ can be identified by successively using the not yet treated at $l+t$ to estimate the exit rate under no treatment at $l+t$ for those treated at $l+s$. Vikström (2014) generalizes by explicitly allowing for selectivity on observables in subsequent assignments into treatment. We follow his approach. Because the identification proof is not affected for different values of $l$, we refer the reader to Vikström (2014).

Before discussing estimation and inference, we first argue why we believe that the available data are sufficiently rich to justify identification on the basis of the $\mathrm{CIA}$, i.e. that we observe all relevant

\footnotetext{
${ }^{26}$ In contrast to e.g. Crépon et al. (2009), Vikström (2014) does not impose $t>s$. This means that he also considers the $\operatorname{ATT}_{s}(s)$, i.e. the treatment effect in the period that the treatment starts. However, identification of this treatment effect requires that the treatment starts instantaneously at the start of this period. Without this assumption the control units are longer at risk of leaving employment than the treated, because treatment is only possible if one survives in employment until the treatment start. This biases the treatment effect upwards. Since we choose the period lengths to be equal to one year, this bias could be substantial.
} 
determinants that influence the decision to participate in TC as well as the survival rate in employment. With regards to the NAA, we refer to our discussion in Section 4.2.

The literature mentions the following key determinants of gradual retirement also affecting the survival in employment: age, household composition, place of living, entitlement to (early) retirement benefits, education, health and firm characteristics, such as size and sector, but also the degree of unionization, organizational features and staff related issues, such as staff and skilled workers shortages (e.g. Gustman and Steinmeier, 1984; Honig and Hanoch, 1985; Huber et al., 2015) . The exhaustive information on the labour market history since 1957 allows us to condition on the most essential information required to determine the level of (early) retirement benefits to which workers are entitled. Household information is sufficiently available. By contrast, the database does not contain information on the level of education and the available indicators of health can only be used as outcomes, not as conditioning variables (see Section 4.2). Nevertheless, we believe that this is not problematic, because health problems should be indirectly captured by gaps in the labour market experience, in lower level of earnings, and being a blue collar worker or not. A similar reasoning applies to the level of education. Huber et al. (2015) stress the importance of having rich firm characteristics to condition upon. However, we believe that this is less important in our institutional environment than in theirs. In Germany firms decide on whether they make the partial retirement available to their employees. By contrast, in Belgium TC is a right to which employees are entitled without consent of the employer, at least in firms larger than 10 employees on which we focus in this analysis. Nevertheless, it is still crucial to condition on the available firm characteristics, i.e. on the firm size and sector, because these are highly correlated with the degree of unionization, and, more relevant for the Belgian context, with the working conditions. This is because working conditions, negotiated in the sectoral industrial committees, usually depend on firm size and apply to all workers, irrespectively of their union membership. Finally, a major pathway to retirement is the bridge pension, so that it is important to control for factors that influence the transition to it. We have seen in Section 3 that the availability of the bridge pension depends importantly on which industrial committee the worker belongs to. Because the number of industrial committees is too large to condition upon in the analysis, we therefore constructed a continuous measure of the propensity of transition to a bridge pension. How this measure is constructed is explained in a footnote to Table 1.

\subsection{Estimation and Inference}

Vikström derives the Inverse Probability Weighting (IPW) estimator, introduced by Horvitz \& Thompson (1952) and Hirano et al. (2003), to estimate the $\operatorname{ATT}_{t}(s)$ defined in (2). We follow this approach for the following reasons: (i) Busso et al. (2014) find in their Monte Carlo simulation that the 
normalized IPW estimator is one of the best performing matching estimators in the presence of good overlap. Other Monte Carlo simulations of (Huber et al., 2013; Frölich et al., 2015) confirm the good performance of the IPW estimator, although it does not outperform other Propensity Score-based and non-parametric estimators; (ii) It is easy to integrate the endogenous sampling weights. This merely requires to include an additional weight in the estimation; (iii) Compared to other matching estimators, the IPW estimator is simple and computationally fast.

We provide the most general estimator that does not only allow to take into account selective (on observables) right censoring as a consequence of not yet treated individuals getting treated (Vikström, 2014), but also more general forms of selective right censoring that may involve both treated and not yet treated individuals. For instance, we will consider estimations of the treatment effect on different exit destinations when terminating employment (i.e. a competing risk framework). We will distinguish between exits to bridge pensions, statutory early retirement and "other" exit routes.

To be able to write down the estimator, let us denote the random censoring duration since sample selection for individual $i$ by $C_{i}$. If we then generalise Vikström's formula (see his Appendix A.3) for the endogenous sampling weights $W_{c r, i}$ and take the elapsed employment duration $l_{i}$ into account, we obtain:

$$
\begin{aligned}
& \widehat{\operatorname{ATT}}_{\mathrm{t}}(\mathrm{s})=\prod_{\mathrm{k}=\mathrm{s}+1}^{\mathrm{t}}\left[1-\frac{\sum_{\mathrm{i}} \mathrm{W}_{\mathrm{cr}, \mathrm{i}} * \mathrm{~W}_{\mathrm{l}, \mathrm{k}(\mathrm{s}), \mathrm{i}}^{\mathrm{C}}(\mathrm{s}) \mathrm{Y}_{\mathrm{k}, \mathrm{i}} 1\left(\overline{\mathrm{Y}}_{1+\mathrm{k}-1, \mathrm{i}}=0\right) 1\left(\mathrm{~S}_{\mathrm{i}}=\mathrm{s}\right) 1\left(\mathrm{C}_{\mathrm{i}}>\mathrm{s}\right)}{\sum_{\mathrm{i}} \mathrm{W}_{\mathrm{cr}, \mathrm{i}} * \mathrm{~W}_{\mathrm{l}, \mathrm{k}(\mathrm{s}), \mathrm{i}}^{\mathrm{C}}(\mathrm{s}) 1\left(\overline{\mathrm{Y}}_{\mathrm{l}}\right)}\right] \\
& -\prod_{\mathrm{k}=\mathrm{s}+1}^{\mathrm{t}}\left[1-\frac{\sum_{\mathrm{i}} \mathrm{W}_{\mathrm{cr}, \mathrm{i}} * \mathrm{~W}_{\mathrm{l}, \mathrm{k}(\infty), \mathrm{i}}^{\mathrm{C}}(\mathrm{s}) \mathrm{Y}_{\mathrm{k}, \mathrm{i}} 1\left(\overline{\mathrm{Y}}_{\mathrm{l}+\mathrm{k}-1, \mathrm{i}}=0\right) 1\left(\mathrm{~S}_{\mathrm{i}} \geq \mathrm{k}\right) 1\left(\mathrm{C}_{\mathrm{i}} \geq \mathrm{k}\right)}{\sum_{\mathrm{i}} \mathrm{W}_{\mathrm{cr}, \mathrm{i}} * \mathrm{~W}_{\mathrm{l}, \mathrm{k}(\infty), \mathrm{i}}^{\mathrm{C}}(\mathrm{s}) 1\left(\overline{\mathrm{Y}}_{1+\mathrm{k}-1, \mathrm{i}}=0\right) 1\left(\mathrm{~S}_{\mathrm{i}} \geq \mathrm{k}\right) 1\left(\mathrm{C}_{\mathrm{i}} \geq \mathrm{k}\right)}\right]
\end{aligned}
$$

where

$$
\begin{gathered}
W_{l, k(s), i}^{C}(s)=\frac{1}{\prod_{m=s+1}^{k}\left[1-c_{m}\left(X_{i}, l_{i}\right)\right]} \\
W_{l, k(\infty), i}^{C}(s)=\frac{p_{s}\left(X_{i}, l_{i}\right)}{1-p_{s}\left(X_{i}, l_{i}\right)} \frac{1}{\prod_{m=s+1}^{k}\left[1-p_{m}\left(X_{i}, l_{i}\right)\right]\left[1-c_{m}\left(X_{i}, l_{i}\right)\right]} \\
p_{t}\left(X_{i}, l_{i}\right)=\operatorname{Pr}\left(S_{i}=t \mid X_{i}, S_{i} \geq l_{i}+t, \bar{Y}_{l+t-1, i}=0\right) \\
c_{t}\left(X_{i}, l_{i}\right)=\operatorname{Pr}\left(C_{i}=t \mid X_{i}, S_{i} \geq l_{i}+t, \bar{Y}_{l+t-1, i}=0\right)
\end{gathered}
$$

where $X_{i}$ denotes the vector of predetermined explanatory variables, $W_{l, k(s), i}^{C}(s)$ and $W_{l, k(\infty), i}^{C}(s)$ are the IPW weights in year $l_{i}+k$ for individual $i$ treated in year $l_{i}+s$ and not yet treated in year $l_{i}+k$, respectively. $p_{t}\left(X_{i}, l_{i}\right)$ and $c_{t}\left(X_{i}, l_{i}\right)$ denote the conditional probability of entering the treatment, respectively censoring state after $l_{i}+t$ years conditional on still being employed in $l_{i}+t-1$. In other words, they represent the discrete hazard of entering treatment, respectively censoring in year $l_{i}+t$. 
To clarify the intuition of the estimator defined in Equation (5) consider first the case without right censoring, i.e. $C_{i}=\infty, W_{l, k(s), i}^{C}(s)=1$ and $W_{l, k(\infty), i}^{C}(s)=\frac{p_{s}\left(X_{i}, l_{i}\right)}{1-p_{s}\left(X_{i}, l_{i}\right)} \frac{1}{\prod_{m=s+1}^{k}\left[1-p_{m}\left(X_{i}, l_{i}\right)\right]}$. Apart from the weights the first sequence of products in (5) is the standard Kaplan Meier survivor estimator for the treated group. This represents the conditional survival rate in employment until year $l_{i}+t$, conditional on treatment and survival in employment until year $l_{i}+s$, i.e. the product of one minus the discrete hazards from employment between $l_{i}+s+1$ and $l_{i}+t$. The second sequence of products is a similar Kaplan Meier estimator for the control group (or not yet treated individuals), which estimates the survival rate of the treated in the counterfactual of no treatment. In order to make these control units comparable to the treated they are reweighted using the standard IPW weights $\frac{p_{S}\left(X_{i}, l_{i}\right)}{1-p_{S}\left(X_{i}, l_{i}\right)}$ in a static evaluation approach, where $p_{s}\left(X_{i}, l_{i}\right)$ is the estimated Propensity Score (PS) for an individual treated in year $l_{i}+s$. However, to take into account that not yet treated individuals gradually become treated, we must consider that this may change the composition of the control group over time. Hence, Vikström (2014) shows that we must in addition weigh the control units by $\frac{1}{\prod_{m=s+1}^{k}\left[1-p_{m}\left(X_{i}, l_{i}\right)\right]}$, i.e. by the inverse of the probability of not yet being treated in each period between $l_{i}+s+1$ and $l_{i}+t$.

If individuals are right censored before exiting to the destination of interest and this is selective (i.e. depends on $X$ ), then this may similarly gradually change the composition of now not only the control group, but also of the treatment group over time. We therefore need to weigh both treated and control samples by $\frac{1}{\prod_{m=s+1}^{k}\left[1-c_{m}\left(X_{i}, l_{i}\right)\right]}$, i.e. the probability of not yet being right censored in each period between $l_{i}+s+1$ and $l_{i}+t$.

In contrast to Vikström (2014), the discrete hazards to treatment and censoring depend on the elapsed employment duration $l_{i}$ at sample selection. ${ }^{27}$ Observe that we can only proxy for this elapsed employment duration, because prior to 1998 we only have annual (instead of quarterly) information on private sector employment and no information on self-employment, neither on employment as civil servant. Given that we selected individuals with at least 5 years of tenure and 20 years of experience in the private sector, we believe that the bias induced by using this proxy is negligible.

We estimate separate ATTs for individuals entering treatment in 2003 and 2004 . Subsequently, we pool, as Vikström, these analyses to have more precise estimates. This is done by averaging the estimated ATTs in each survival year, taking into account the size of the two different treated groups in the population, and, hence, weighted by the endogenous sampling weights.

\footnotetext{
${ }^{27}$ Because the Kaplan Meier estimators in Equation (6) are averaged over all individuals in the sample, we average, as in the definition of the ATT in Equation (2), implicitly over the elapsed employment durations, conditional on this elapsed duration to exceed 5 years.
} 


$$
\widehat{\operatorname{ATT}_{t}}=\sum_{s} \frac{n_{s}}{\sum_{s} n_{s}} A \widehat{T_{T}+t}(s)
$$

where $n_{s} \equiv \sum_{i} W_{c r, i} * 1\left(S_{i}=s\right)$.

As the lack of overlap of the PS can bias the estimator and increase the variance (Lechner and Strittmatter, 2014), we trim treated units with too high PS not having a correspondent control unit in a neighbourhood. In particular, we remove the treated units with a PS above the 99.9 percentile of the control units. After trimming, we remain with about $99 \%$ of the treated units, counting 1,212 and 755 men and women. (Huber et al., 2013) propose to remove the control units with a weight higher than $4 \%$ of the total. However, because the sample of control units is large, this additional trimming is not required. In the four analyses (2003 and 2004, men and women) the highest relative weight is only $0.17 \%$ of the total sample.

By weighing, the estimator becomes heteroskedastic. In addition, an individual in the 2003 sample may appear a second time in the 2004 sample. To take the resulting correlation in account, we cluster the standard errors by individual. However, standard clustering does not take into account that the PS in the weights $W_{l, k(.), i}^{C}(s)$ are estimated. We therefore bootstrap the standard errors. Since our data come from an endogenously stratified sample, we cannot apply a standard bootstrap. Instead, we implement a stratified bootstrap by randomly drawing for each replication $n_{c r}$ individuals within each cohort-stratum $\mathrm{cr}$. This is valid because the bootstrap randomly samples individuals within each cohort-stratum (for a review on bootstrap and stratified data see e.g. Shao, 2003). To take individual serial correlation into account we re-sample within each replication the same individuals (i.e. clusters) in the two analyses (2003 and 2004 sample).

In general, based on observables, the selectivity into treatment is low in 2003. The Pseudo R-squared of a standard logit model is 0.068 and 0.026 for men and women. The selectivity is slightly higher in 2004. The corresponding Pseudo R-squared are 0.127 and 0.084 . In terms of specification, the IPW estimator performs well in balancing the distribution of the covariates. Once reweighting the control units by $W_{l, 1(\infty), i}^{C}(s)$, in the worse scenario (women selected in 2004) the median Standardized Bias (SB) is as low as $1.2 \%$, the highest SB is $2.9 \%$, the Pseudo R-squared of the reweighted sample is 0 and the Wald test ${ }^{28}$ for the joint significance of the variables after the reweighting produces a pvalue of 1. In Appendix C, we report the full list of balancing tests (Table C.3). The corresponding diagnostics for the other models such as heterogeneous effects can be obtained from the authors on request.

\footnotetext{
${ }^{28}$ The Wald test should be used to assess the balancing as the log-likelihood ratio test proposed by Sianesi (2004) is not robust against heteroskedasticity and serial correlation. It tends to over-reject the null of good balancing.
} 


\section{Results}

We first report the $\widehat{\mathrm{ATT}_{t}}$ from the first until the eight year after entry in the TC $(t \in\{1, \ldots 8\})$ on the main outcome of interest. In Section 3 we have argued that, relative to full-time workers, the TC scheme increases the incentives to enter early retirement, in particular the early statutory retirement scheme from the age of 60 . Moreover, we argued that these incentives were more important for participants in the $50 \%$ TC regime than those in the $20 \%$ regime. In Section 6.2 , we demonstrate that the empirical evidence is in line with these incentives. To that end we estimate the different $\overline{A T T}_{t}$ of each TC regime separately for the three following exit destinations of employment: bridge pension, statutory early retirement and other exits. In this section we also split the sample according to age to study treatment heterogeneity for the benchmark outcome. Finally, in Section 6.3 we study the effect of the TC on the health conditions of participants. We do not consider sick leave as a separate exit destination, because the number of exits to this destination is too small for credible inference. Instead, we broaden the benchmark outcome by including temporary sick leave into failure risk and compare the estimates to the ones considering non-employment (the benchmark) as the only exit destination. All the analyses are conducted separately for men and women. The reported results pool the 2003 and 2004 sample.

\subsection{The Benchmark Analysis}

In this section we show the estimates of the ATT on the survival rate in employment controlling for selection on observables (Vikström, 2014). As Figure 3 shows, estimates are not significantly different from the descriptive evidence shown in Section 4.3. Treated men are more likely to survive in employment in the first two years ( $+7 \mathrm{pp}$ and $+3.5 \mathrm{pp}$ ), while for women the positive effects last until the fourth year and are stronger $(+8.8 \mathrm{pp},+10.9 \mathrm{pp},+8.6 \mathrm{pp}$ and $+3.2 \mathrm{pp})$. The more positive effect for women is partly, but not completely, explained by the fact that they are less likely to work in industrial committees that intensively use bridge pensions as a way to terminate employment early, especially in the $20 \%$ regime (see below). However, the results also confirm the strong subsequent negative effects, peaking up to $-20 \mathrm{pp}$ and $-12 \mathrm{pp}$ for men and women, respectively. Notice that as a consequence of ageing all individuals, both treated and non-treated, will eventually retire. This explains why the treatment effect eventually always tends to zero at the end of the period of analysis. From these estimates, it seems that controlling for the rich set of covariates does not significantly affect the differences in survival rates between the treated and the control groups. This is because the imposition of the TC eligibility conditions on the control group has already enhanced the comparability of treated and control groups very much. 
Figure 3: ATT on Survival in Employment and Descriptive Evidence

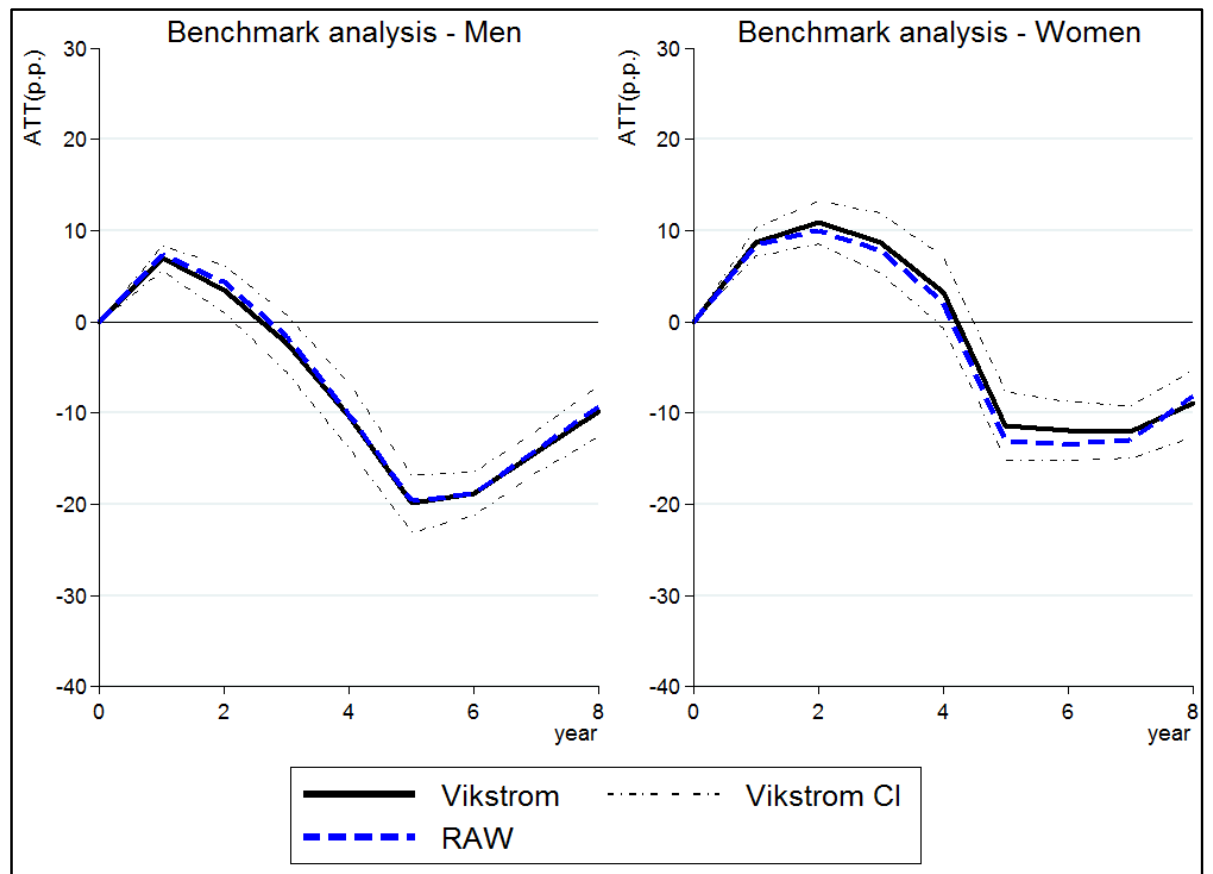

ATT on the survival rate in employment estimated by controlling for the dynamic selection on observables (Vikström, 2014) and descriptive evidence (RAW) as reported in Figure 1. The estimates of the ATT's are the percentage points (pp) differences between the survival rate of the treated in case of treatment and the estimated survival rate of the treated in the counterfactual of no treatment. Estimates are pooled over the 2003 and 2004 samples. Year eight only uses information from the 2003 sample. Standard errors are obtained by a stratified bootstrap (clustering by individual) with 500 repetitions and $95 \%$ confidence intervals $(\mathrm{Cl})$ by assuming normality.

Our results are in line with the findings of Graf et al. (2011) in Austria. Similarly to the Austrian scheme, our estimates show that individuals entering the schemes tend to remain in employment for a few years longer. However, the cumulative impact becomes negative after some years. The positive effects in the beginning are likely present because, as long as they are not eligible to early retirement, the TC participants keep on working part-time while accumulating pension rights of a full-time worker. However, as these workers also start to satisfy the age and experience requirements, they gradually enter the most remunerating early retirement scheme. To gain more insights into the forces driving these results, we decompose in Section 6.2 the ATTs by distinguishing between the TC-regime (20\% or $50 \%)$, the destination-specific survival rates in employment and the age of the participants.

\subsection{Competing Exit Destinations and Different Treatment Regimes}

In this section we decompose the estimated ATT on the survival in employment according to three possible exit destinations: (i) bridge pension, (ii) statutory early retirement and (iii) other exits. The last is a residual category comprising other schemes such as Canada Dry, unemployment, disability and other forms of inactivity, and exit because the individual deceased. As to simplify the estimation procedure, we do not estimate these competing risks simultaneously, but right censor the other destinations, when considering the destination of interest. This is valid because, conditional on the 
observed covariates, the latent destination specific survival times are independent of each other. Recall that we have shown in the descriptive analysis in Section 4.3 that most of the exits are to the first two destinations. For a better understanding of the role of financial incentives, we divided the treated sample into participants in the 50\% (Figure 4) and 20\% TC-regime (Figure 5). The treated sample is divided in 942 units participating in the 50\% TC-regime and 1,047 in the $20 \%$ regime. In the corresponding population $54.3 \%$ of TC participants are in the $50 \%$ regime. Note that the same control units are used for estimating the ATTs of these two treatment groups. Estimates of the competing risks for the whole sample of participants irrespectively of the TC-regime are shown in Figure 6, which also displays the sensitivity of the ATTs to the estimation method. To have an idea of the relative size of the ATT we report the survival rates of the treated and (reweighted) control units in Figure C.1 of Appendix C. As these people are more intensively treated, the positive ATT in the short-run and the negative ATT in the medium-run are also more pronounced compared to the participants in the $20 \%$ regime. As treated individuals remain entitled to the statutory (early) pension as full-time workers, their replacement rate is much higher than that of control individuals. This explains the strong response on the survival in employment without exit to statutory early retirement. Once we right censor these exits to statutory early retirement, the effect for TC participants with the $50 \%$ reduction is non-negative for the other two exit destinations. The treated individual working at $80 \%$ of a full-time have a less pronounced response, especially for the exit to statutory early retirement, and rather show more noticeable differences by gender (Figure 5). Different from the $50 \%$ regime, we now also observe for men a negative impact on the survival in employment without exit to the bridge pension, while for women the impact is insignificant.

As described in Section 3, while the bridge pension is in general very appealing for older workers in Belgium, it is not for workers in the 50\% TC-regime, because they lose a large part of the benefits. However, because this loss is less important for individuals in the $20 \%$ regime, the scheme still remains attractive for them. This incentive does not affect the behaviour of women, however, because they are on average less likely to work in industrial committees that intensively use the bridge pension as instrument to terminate employment (see Table 1), and, hence, have less opportunities to use this pathway to retirement. More generally, because women tend to have acquired slightly less labour market experience (and have lower past earnings) than men (Table 1), they are less likely to be eligible for (and earn a decent income when entering) early retirement which may explain their better overall effects of TC on the survival rate in employment. 
Figure 4: ATT of Treated Men (A) and Women (B) in 50\% TC Scheme - Competing Risk (CR) and Baseline (non-CR) Framework
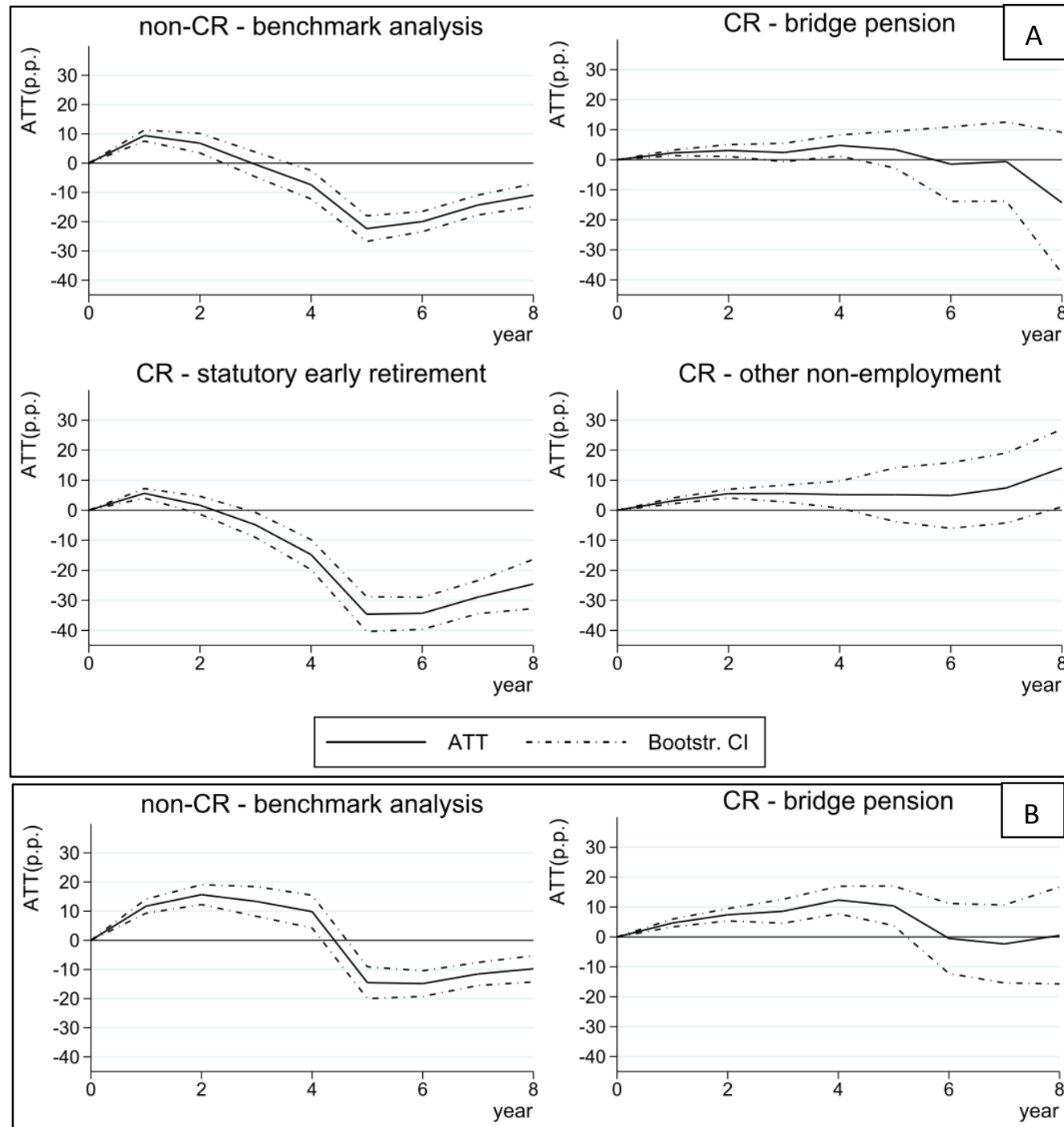

Bootstr. Cl
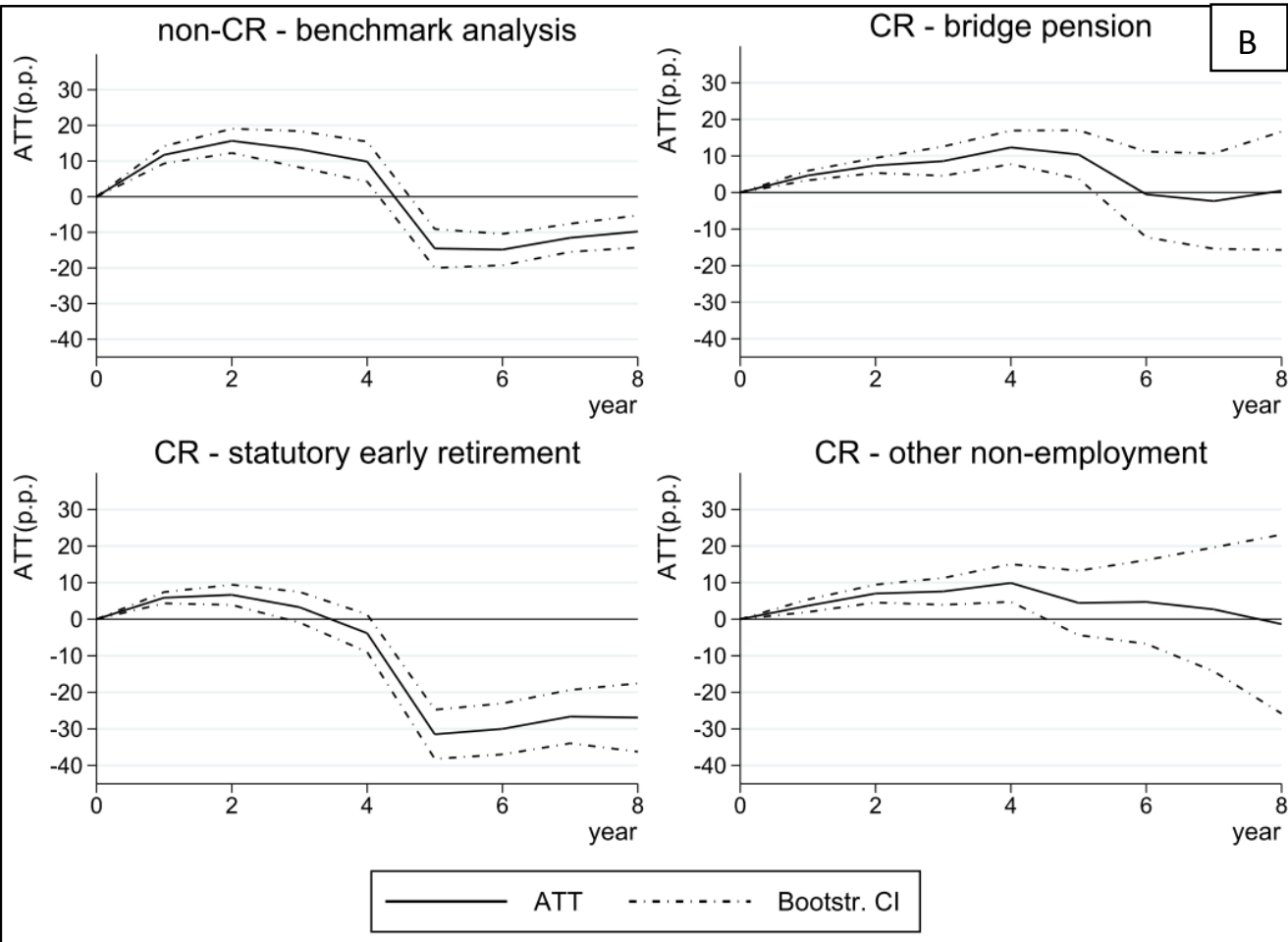

ATT of treated in the $50 \%$ TC on the survival rate controlling for the dynamic selection on observables (Vikström, 2014). The ATTs are differentiated by gender: Panel A for men and B for women. The estimates are expressed in percentage points (pp) differences in the survival rate in (from left to right and top to bottom) (1) employment, (2) employment without exit to a bridge pension, (3) employment without exit to a statutory pension before the normal retirement age ( 65 for men and women born after 30/11/1944, 64 for women born between 01/12/1942 and 30/11/1944 and 63 for women born before $30 / 11 / / 1942$ ) and (4) employment without exit to other non-employment statuses. In the competing risk analyses (2-4), the exits from employment to other destinations, apart from the one considered, are right censored. Reported estimates are pooled over the 2003 and 2004 samples. Year eight only uses information from the 2003 sample. The pooled sample is composed of $567(375)$ treated and 29,791 $(9,658)$ control units (men and women). Standard errors are obtained by a stratified bootstrap (clustering by individual) with 500 repetitions and $95 \%$ confidence intervals $(\mathrm{Cl})$ by assuming normality. 
Figure 5: ATT of Treated Men (A) and Women (B) in 20\% TC Scheme - Competing Risk (CR) and Baseline (non-CR) Framework
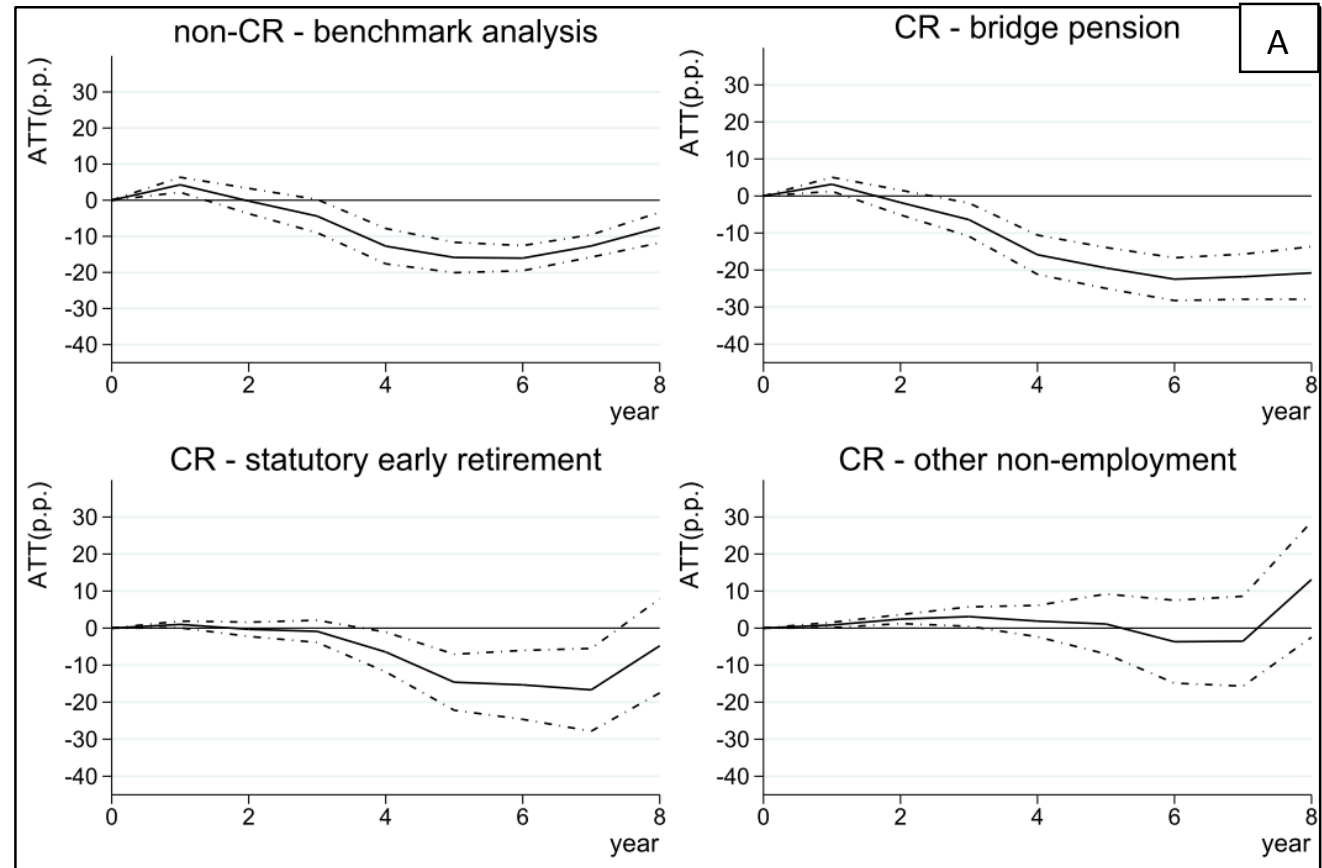

ATT $\quad \cdots \cdots$ Bootstr. Cl

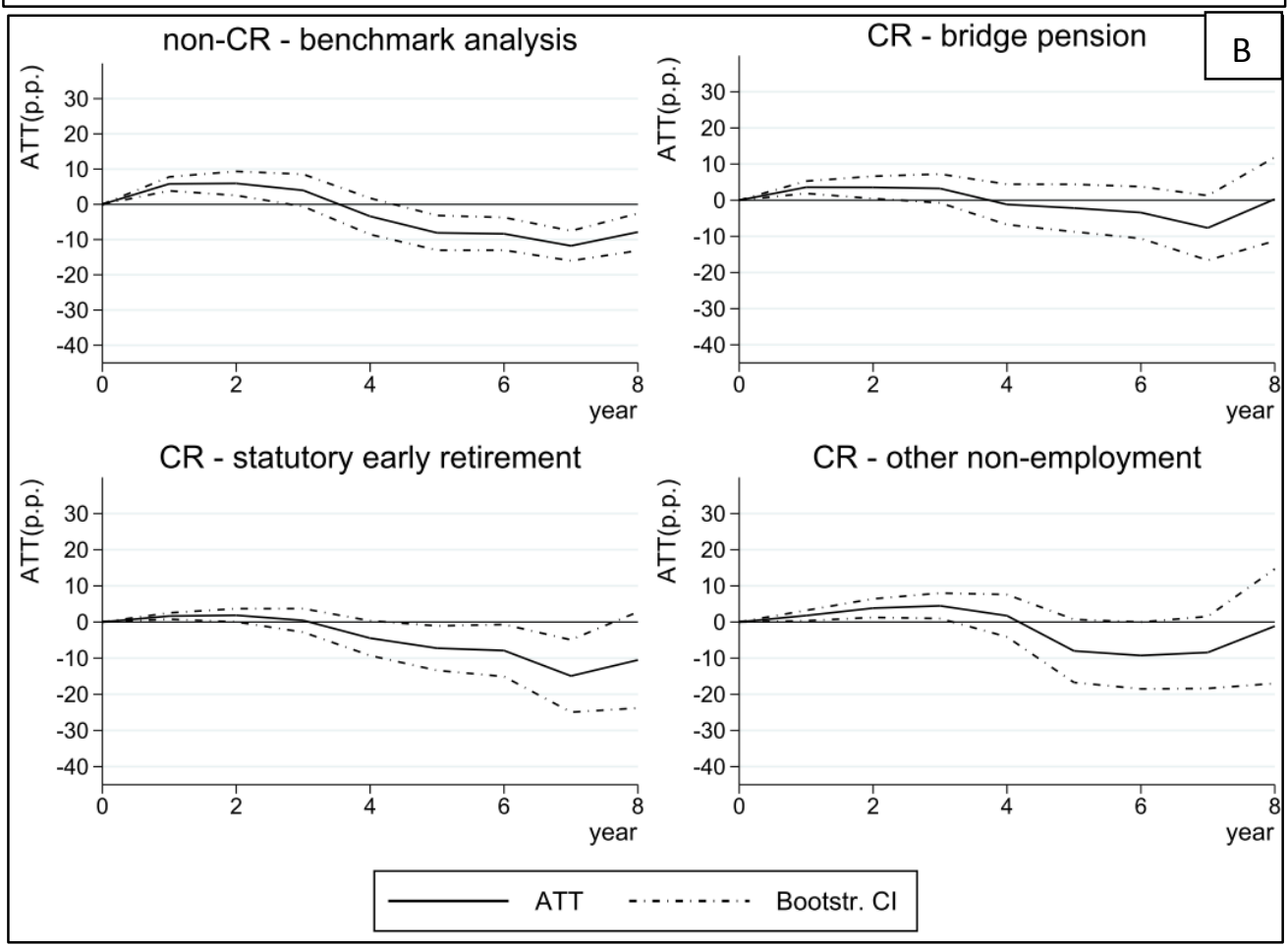

ATT of treated in the $20 \%$ TC on the survival rate controlling for the dynamic selection on observables (Vikström, 2014). The ATTs are differentiated by gender: Panel A for men and B for women. The estimates are expressed in percentage points (pp) differences in the survival rate in (from left to right and top to bottom) (1) employment, (2) employment without exit to a bridge pension, (3) employment without exit to a statutory pension before the normal retirement age (65 for men and women born after 30/11/1944, 64 for women born between 01/12/1942 and 30/11/1944 and 63 for women born before $30 / 11 / / 1942)$ and (4) employment without exit to other non-employment statuses. In the competing risk analyses (2-4), the exits from employment to other destinations, apart from the one considered, are right censored. Reported estimates are pooled over the 2003 and 2004 samples. Year eight only uses information from the 2003 sample. The pooled sample is composed of $660(387)$ treated and 29,791 $(9,658)$ control units (men and women). Standard errors are obtained by a stratified bootstrap (clustering by individual) with 500 repetitions and $95 \%$ confidence intervals (CI) by assuming normality. 
Next, as we argued that eligibility to early retirement affects the impact of the TC scheme, we split the sample between younger and older individuals. We set the cut-off age at 56.5 years at the moment of sample selection, i.e. at the end of year 0 . In theory, the younger workers are too young to already be eligible to any of the early retirement schemes. Thus, we expect a longer-lasting positive effect for them. As shown in Figure C.3 in Appendix C, this is indeed what we find and corroborates therefore our interpretation of the findings. ${ }^{29}$

Finally, we studied to what extent it matters for our results to take the dynamic assignment into treatment into account (Fredriksson and Johansson 2008) and to, in addition, control for selective (on observables) right censoring (Vikström, 2014). In Figure 6 we therefore compare our estimates based on Vikström's (V) methodology to the descriptive estimator (raw data) on the one hand and to the estimator proposed by Fredriksson and Johansson (FJ) on the other hand. While in the descriptive evidence we ignore dynamic assignment to treatment in that we do not remove (i.e. exogenously right censor) controls becoming treated and do not control for differences in observables, we do when implementing the FJ estimator by IPW.

We deduce the following two observations from Figure 6. First, the raw estimates are not very different from the ones that take the dynamic assignment into treatment into account (FJ), except for the survival in employment without exit to statutory early retirement in which case the raw estimates are downwards biased. Second, the FJ estimator is significantly downwards biased relative to the V estimator for the survival in employment without exit to the bridge pension and without other exit.

These observations lead to the following conclusions. First, in the benchmark model (without competing destinations) the estimates are not sensitive to the employed estimator. This is because the selection on observables is not important and the bias induced by the dynamic assignment to the treatment is small, as only a small fraction of the not yet treated group enters into treatment later on. Second, the estimation method matters more when analysing competing risks, because in this analysis the fraction that is right censored (in both treatment and control groups) is much more important than the dynamic assignment into treatment. If the right censoring is selective on observables, which is clearly the case for exits to the statutory early retirement, ${ }^{30}$ then this bias can only be avoided by using the $\mathrm{V}$ estimator.

\footnotetext{
${ }^{29}$ We have also estimated treatment heterogeneity with respect to labour market earnings at selection. The results are very similar to the different response by TC regime as two thirds of the treated high earnings group take the $50 \%$ regime (symmetric figures for the low earnings group). Results are available from the authors upon request.

${ }^{30}$ Since exits to the statutory early retirement are treated as right censored observations for the other two destinations, this explains the second observation that we deduced from Figure 6. The fact that the effect on the survival rate in employment without exit to the statutory early retirement does not differ very much using either the V or FJ estimator suggests that exits to the aforementioned other two destinations are not very selective. On the other hand, the fact that these estimates do differ from the raw estimates reflects again that exits to this destination are selective on observables.
} 
Figure 6: ATT of Treated men (A) and Women (B) and Comparison with Other Estimators
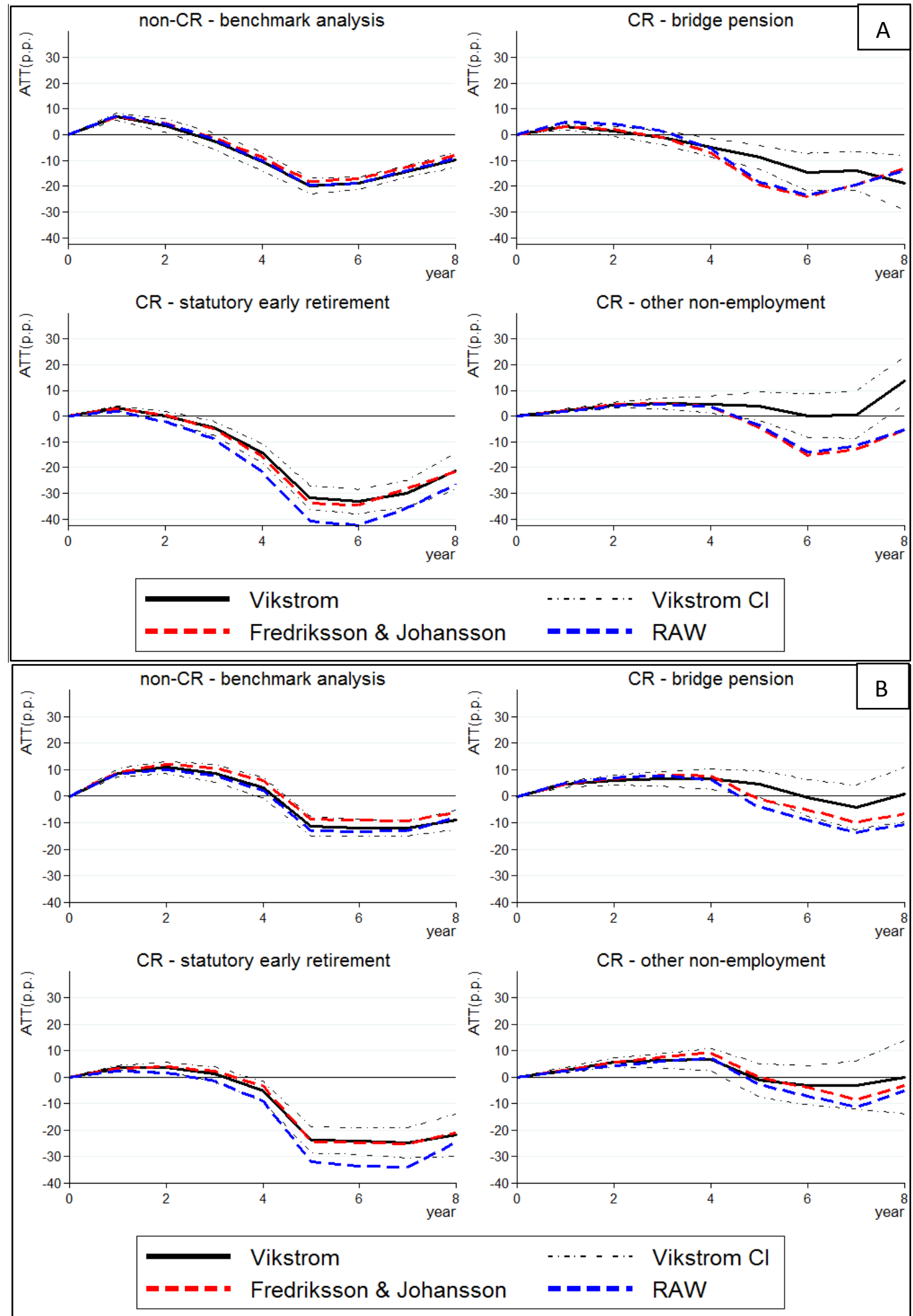

Vikström: ATT of treated on the survival rate controlling for the dynamic assignment to treatment \& selective right-censoring on observables (Vikström, 2014); Fredriksson \& Johansson: controlling for selection on observables in the year of selection (2003 or 2004) but not for selective right-censoring on observables (Fredriksson and Johansson, 2008); RAW: neither controlling for selection on observables, nor on dynamic assignment to treatment. The ATTs are differentiated by gender: Panel A for men and B for women. The estimates are expressed in percentage points ( $\mathrm{pp}$ ) differences in the survival rate in (from left to right and top to bottom) (1) employment, (2) employment without exit to a bridge pension, (3) employment without exit to a statutory pension before the normal retirement age (65 for men and women born after 30/11/1944, 64 for women born between 01/12/1942 and 30/11/1944 and 63 for women born before 30/11/1942) and (4) employment without exit to other non-employment statuses. In the competing risk analyses (2-4), the exits from employment to other destinations, apart from the one considered, are right censored. Reported estimates are pooled over the 2003 and 2004 samples. Year eight uses information from the 2003 sample. The sample is composed of 1,227 (762) treated and 29,791 (9,658) control units (men and women). Standard errors are obtained by a stratified bootstrap (clustering by individual) with 500 repetitions and $95 \%$ confidence intervals (CI) by assuming normality. 
In conclusion, our estimates suggest that older workers use part-time work at the end of the career as an alternative path to (early) retirement. This enables them to continue working until they become eligible to early retirement schemes. At that moment TC participants have much higher incentives to enter these schemes than non-participants. Moreover, the evidence is consistent with participants acting upon these incentives, since they not only leave employment faster than non-participants, but they also choose the exit channel that offers them the highest replacement income. We therefore believe that these supply side incentives matter more than other explanations advanced in the literature for these negative effects on the survival rate in employment, such as the higher hourly workload (Devisscher and Sanders, 2007; Rudolf, 2014), the TC signaling a preference to retire (Machado and Portela, 2012), or the reduced working time decreasing the labour market attachment.

\subsection{Including Sick Leave as Additional Exit Destination}

It has been argued that granting working time reductions can reduce the incidence of sickness (Ahn, 2015). In this section we aim at testing this hypothesis by checking whether TC recipients are less likely to enter sick leave (while employed) than non-recipients. A first observation is, however, that few individuals in our sample do enter sick leave. In the control group the fraction is $14.8 \%$, while in the treated group it is $13.0 \%$. This is because our sample selection criteria exclude people with fragile health. More specifically, by imposing that workers should have at least five years of tenure in a firm, we exclude individuals who have temporarily interrupted employment during a full quarter, while including individuals who have been on sick leave throughout this quarter (Section 4.2). Because the fraction of individuals entering sick leave is relatively small, the impact of TC on this indicator of health cannot be large. Nevertheless, we attempted to estimate the ATT on sick leave to get a sense of the direction of the effect.

Since so few individuals entered sick leave, we did not consider sick leave as a separate exit destination in the analysis, because the sample would become too small. Instead, we include sick leave as an additional exit destination, so that we estimate the impact of the TC on being employed and in good health. We then compare this treatment effect to the one we obtained for the benchmark model (Section 6.1). In Figure 7 we report these two treatment effects. We observe that the new treatment effect (dashed line) always exceeds the benchmark (solid line). This suggests that TC reduces the incidence of sick leave. However, the dashed line is mostly comprised in the $95 \%$ confidence interval of the benchmark ATT, suggesting that the effect is not statistically significant. 
Figure 7: ATT on the Survival in Employment \& Employment and Not on Sickness Leave

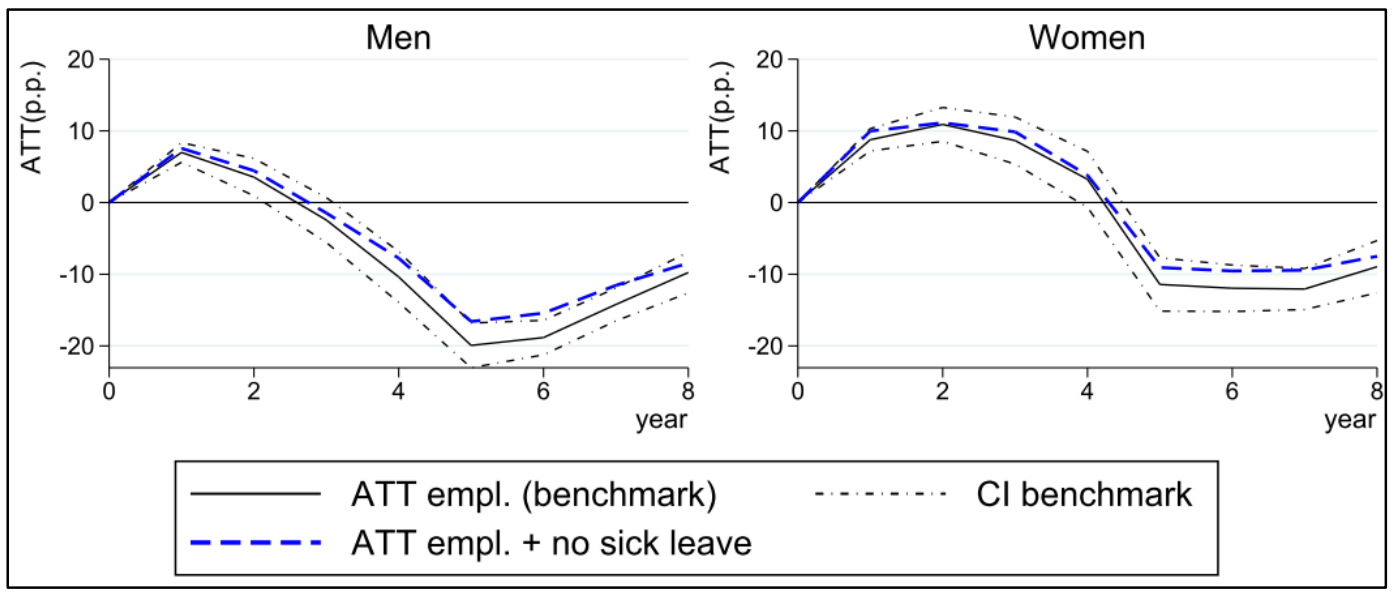

ATT on the survival rate estimated by controlling for the dynamic selection on observables (Vikström, 2014). The survival is in employment (ATT empl.) or in employment while not on sick leave (ATT empl + no sick leave). The estimates are expressed in percentage points (pp). Estimates are pooled over the 2003 and 2004 samples. Year eight only uses information from the 2003 sample. The pooled sample is composed of 1,227 (762) treated and 29,791 $(9,658)$ control units (men and women). Standard errors are obtained by a stratified bootstrap (clustering by individual) with 500 repetitions and $95 \%$ confidence intervals $(\mathrm{Cl})$ by assuming normality The $\mathrm{Cl}$ reported are referred to the benchmark scenario having employment as the outcome (ATT empl benchmark).

\section{Cost-Benefit Analysis}

To obtain an order of magnitude of the costs and benefits of the TC for the government budget and for society, we perform a cost-benefit analysis (CBA) along the lines proposed by Staubli and Zweimüller (2013). To that purpose we make use of the information available in the administrative dataset on the benefits and gross wages that are paid out to participants and non-participants in the TC. This information is then used to calculate for each individual and in each of the 8 years of analysis after the year of (counterfactual) entry in TC the real costs (or gains) in constant 2004 Euros for the government budget and for society. We weigh the control group by the appropriate IPW to make them comparable to the treated group and calculate for each of these years the average difference of these costs (gains). This provides an estimate of the average net cost (gain) per participant in TC for the government and society in each of these eight years (Staubli and Zweimüller, 2013; Albanese and Cockx, 2015). Differently from the ATT on the survival in employment, these have to be interpreted as the instantaneous net costs (gains) during those years.

In the literature review we mentioned that Huber et al. (2015) found that in East-Germany participating women in the gradual retirement scheme were partially replaced by unemployed younger women. Whether this replacement occurs or not matters a lot for the CBA. Our data do not allow to test this hypothesis though. Nevertheless, we consider two scenarios in the CBA. One assumes that the part-time work is not replaced by another part-time worker, the other assumes that this parttime work is replaced by another who earns an equivalent wage and is equally productive as the part- 
time worker. In this second scenario we do not take into account, however, the gains in terms of UB payments that would no longer have to be paid if the replacing worker came from unemployment.

There are a number of reasons why we cannot perform a full-fledged CBA. First, as we will explain more in detail below, not all required information at the individual level is available in the administrative database. In these cases we substitute the individual level information by aggregate information obtained from other sources or, if it refers to a very small share of individuals, we ignore the information by setting it to zero. Second, the analysis ignores some important dimensions. For instance, in Section 6.3 we found some limited evidence that participation in the TC may have some small positive health effects. However, as we lack information on health costs, we cannot take this dimension into account. Moreover, we ignore the impact of participation in TC on the distribution of welfare or on poverty. All this means that the CBA should be taken as a crude approximation.

\subsection{Methodology}

We calculate, for each year $t$ of the period of analysis above, the effect of the policy on two indicators, the Net Budgetary Cost for the Government (NBC) and the Net Welfare Cost for Society (NWC). Both indicators are expressed per treated individual and in monthly terms (2004 Euros).

i. Net budgetary cost (gain) for the Government (NBC): This is the average cost (gain) of the policy for the state, net of savings for the public budget:

$$
N B C_{t}=\text { allow }_{t}-\operatorname{tax}_{t} * \operatorname{remu}_{t}-S S C_{t} \text {, }
$$

with:

- allow $_{t}$ : expenditures of the Government on allowances of the Social Security scheme, such as unemployment benefits and the TC allowance. Because the database lacks information on statutory pension, sickness and disability allowances, we impute these allowances as follows:

- We assume that the worker has worked his entire career in the private sector and assume that the individual is paid the average pension in the private sector according to the age bracket to whom he belongs: 50-54, 55-59, 60-64, 65-69.

- For sickness and disability benefits we set the allowance equal to the theoretical level of entitlement, i.e. to $60 \%$ of the individuals' average monthly remuneration over the last six quarters.

- $\operatorname{tax}_{t}$ : The average personal income tax rate on the gross remuneration in every year (OECD, 2015a).

- remu $_{t}$ : the gross wage earnings. This is observed in the data for employees in the private and public sector, but not for the self-employed, for whom we impute a zero value for both treated 
and control units. Since the share of self-employed individuals in the control group is larger (4.6\%/2.0\% of men / women) than in the treated group (2.0\%/0.7\% of men / women), this slightly biases our cost estimate downwards.

- $S S C_{t}$ : employer and employee contributions to Social Security.

ii. Net Welfare cost (gain) for Society (NWC): the efficiency cost ("excess burden" or "deadweight loss") of the net budgetary expenditures mentioned in (i) plus the opportunity cost of working minus the production value of employment (PV):

$$
\begin{aligned}
N W C_{t} & =(M C F-1) * N B C_{t}+L E I S_{t}-P V_{t} \\
& =(M C F-1) * N B C_{t}+L E I S_{t}-\left(1-P G_{t}\right) * L C_{t}
\end{aligned}
$$

with:

- $\quad M C F$ : the Marginal Cost of Public Funds. ${ }^{31}$ For Belgium a MCF equal to two is considered to be appropriate (Kleven and Kreiner, 2006; Barrios Cobos et al., 2013).

- $\quad L E I S_{t}$ : the opportunity cost of working, which has to be between zero and the net wage plus the SSC). In the latter we include both employee's and employer's Social Security contributions as they can be seen as an insurance premium to entitlements to Social Security benefits. Similarly to Greenberg and Robins (2008), we use the mid-point between the two bounds as our benchmark estimate.

- $\quad P G$ : the age-related pay-productivity gap takes into account that the wage cost of older workers exceeds their productivity. An estimate of the production value of labour (PV) is obtained by downward adjusting the labour costs $(L C)$ by this gap. We use estimates of this pay-productivity gap provided by Vandenberghe et al. (2013) for the Belgian case.

We consider a sensitivity analysis in two directions. First we consider two scenarios: one in which the effect of reduced working time on labour costs is taken into account and one in which it is not (i.e. full replacement of the reduced working hours for both treated and controls). Second, we check the robustness of our results by varying three key parameters of our model:

1. Two personal income tax rates: for persons earning $100 \%$ (benchmark) or $133 \%$ of the average wage.

2. Three marginal costs of public funds: 1.41, 2.14 (benchmark) and 3.23 (Kleven and Kreiner, 2006).

\footnotetext{
${ }^{31}$ The net budgetary cost is in se not a cost to Society as it just involves transfers between individuals.
} 
3. Three values for the opportunity cost of working: the aforementioned lower and upper bound, as well as the midpoint (benchmark).

In order to measure the impact of TC on these indicators, we proceed in the following way. We run by gender a pooled weighted regression on all 8 years of analysis $(t \in\{1,2, \ldots 8\})$ separately for $s \in\{0,1\}$, i.e. the two samples of analysis:

$$
\sqrt{W_{i t}^{R}} Y_{i t}=\sum_{t=s+1}^{8}\left(\alpha_{t}+\beta_{t} 1\left(S_{i}=s\right)\right) \sqrt{W_{i t}^{R}} D_{t}+\sqrt{W_{i t}^{R}} u_{i t}
$$

with

$$
W_{i t}^{R} \equiv W_{c r, i}\left[1+\frac{p_{s}\left(X_{i}, l_{i}\right)}{1-p_{s}\left(X_{i}, l_{i}\right)} \frac{1}{\prod_{m=s+1}^{t}\left[1-p_{m}\left(X_{i}, l_{i}\right)\right]} 1\left(S_{i}>t\right)\right]
$$

where $Y_{i t}$ measures the outcome of interest, i.e. NBC or NWC, for each individual $i$ in year $t$ after treatment assignment, $D_{t}$ is a year indicator equal to one in year $t$ and zero otherwise, $u_{i t}$ is the error term of the regression. In this regression $\alpha_{t}$ measures the average outcome for the control units that have not yet been treated in year $t$, i.e. $S_{i}>t>s$, so that $1\left(S_{i}=s\right)=0$, while $\beta_{t}$ measures the average treatment effect on the treated in year $t\left(A T_{t}\right)$, i.e. $S_{i}=s<t$, so that $1\left(S_{i}=s\right)=0$. The weights $W_{i t}^{R}$ ensure that (i) the endogenous sampling is taken into account by weighing all individual observations by $W_{c r, i}$, (ii) the control units that have not yet been treated are made, respectively kept comparable to the treated units by weighing them by the standard IPW $\frac{p_{s}\left(X_{i}, l_{i}\right)}{1-p_{S}\left(X_{i}, l_{i}\right)}$ and by $\frac{1}{\prod_{m=s+1}^{t}\left[1-p_{m}\left(X_{i}, l_{i}\right)\right]}$ to take the selective assignment into treatment over time into account. Note, in contrast to the analysis on the survival rate in employment, individuals who leave employment are not dropped from the analysis. Only individuals in the control group who become treated are dropped, but the weights avoid that this induces selectivity in the comparison between treated and control units. For each time period $t \in$ $\{1,2, \ldots 8\}$ we can then estimate the $A T_{t}$ on $N B C$ or on $N W C$ for each treatment group $s \in\{0,1\}$ by $\hat{\beta}_{t-s}$, where the hat denotes the estimate of the weighted regression in (10). Subsequently, we average over treatment groups in a similar way as in (6).

\section{2. $\quad$ Results}

The results of the benchmark cost-benefit analysis (CBA) can be found in Figure 8, which shows the monthly cost per treated for the government budget and the welfare cost to society. Both the scenarios with and without replacement of part-time workers are discussed.

Without the replacement of part-time workers, the results indicate that the TC scheme is an expensive policy that fails the cost-benefit test. Although in the first years we have estimated a positive employment effect, the costs of the policy immediately dominate the benefits. In the first year of the 
analysis the monthly costs to the government budget in the baseline case are $€ 878$ (€696) per treated person for men (women). That these budgetary costs for the government already exist from the first years indicate that the positive effects on employment at the extensive margin are immediately dominated by the reduction of working hours and the allowance paid to the TC participants. In particular, (i) treated people reduce their working hours, resulting in less income for the government in the form of taxes; (ii) TC participants receive the TC benefit, resulting in higher expenditures for the government. As a reduction in working hours also implies lower production, the monthly total welfare costs per treated individual are even higher: $€ 1,706(€ 1,345)$ for men (women). The costs to society show a decreasing pattern, while near the end of the analysis we observe a stronger decrease for both the costs to the government and the society. As already mentioned, this convergence is induced by the fact that all individuals eventually retire, irrespectively of treatment, so that costs converge to zero. If full replacement of the reduced working hours is assumed, a small short-run positive welfare gain for society emerges, but only for women. This is because in this scenario no working hours are lost when a participant enters the TC scheme. To the extent that there are positive employment effects as discussed in Section 6.1, this reduces the negative budgetary impact, while the net gain for society also comes from the positive impact of the TC scheme on the value of leisure. The largest potential benefits are obtained at the start of the analysis and are larger and longer-lasting for women, as they have more favourable short-run employment effects. ${ }^{32}$ The welfare cost to Society is about zero in year two and four, respectively for men and women. At those points the ATTs on the survival rate in employment was estimated to be about $3.5 \mathrm{pp}$. This suggests that a positive ATT of about 3.5 percentage points is required to break-even in terms of social welfare. However, this is measured for the most optimistic scenario. In reality it is unlikely that employers could replace all reduced working time.

In Appendix C we included two summary tables (Table C.1 and Table C.2) containing the effects for our sensitivity analyses in which we consider all possible scenarios. Though the magnitude of the estimates in the sensitivity analysis changes, the qualitative results are in line with the findings of the baseline scenario. Our CBA ignores distributional effects and effects on other channels such as health. However, it is clear that without full replacement, the policy fails the cost-benefit test.

\footnotetext{
${ }^{32}$ There are almost no benefits for the government budget, while the monthly welfare benefits for society are larger in year one (two) for men (women), when they amount to $€ 80$ (231) per treated man (woman). Similar to the ATT on survival in employment, the period with a welfare gain lasts only one year for men, while three years for women.
} 
Figure 8: Monthly Cost of the TC per Treated of 2003 (2004)

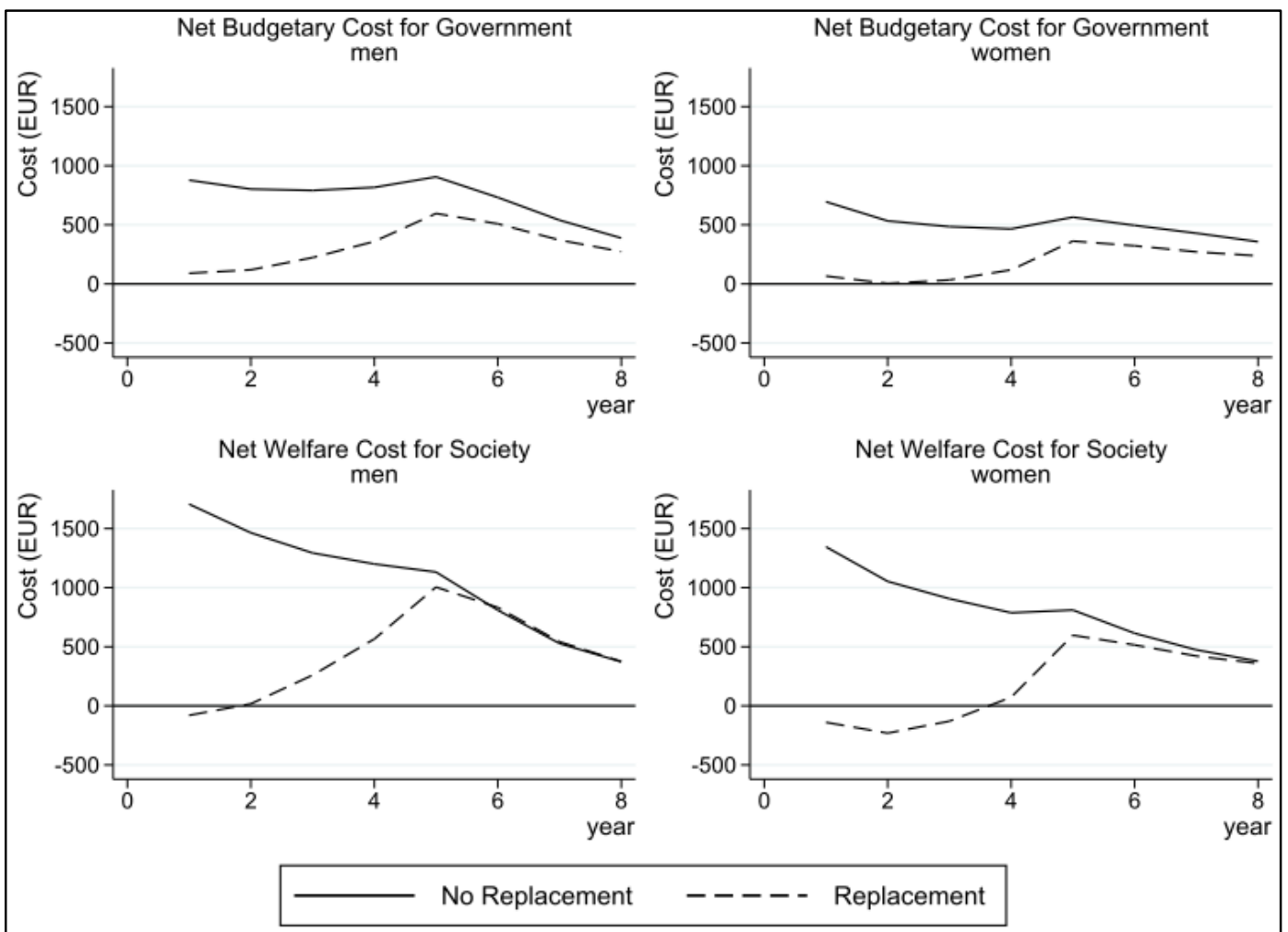

Cost-benefit analysis (CBA) on the pooled sample of participants in TC of 2003 and 2004. CBA in monthly costs (benefits if negative) in 2004 euros per treated individual (the size of the treated sample as defined in 2003/2004). The Net Budgetary Cost (NBC) for the government is the average cost (gain) of the policy for the state, net of savings for the public budget. The Net Welfare Cost (NWC) for society is the efficiency cost of the NBC minus the production value of employment (PV). Baseline scenario: CBA without replacement of the part-time workers. Replacement scenario: baseline scenario with the additional assumption that all hours reduced by part-time workers (treated and controls) are recovered by hiring extra workers with similar characteristics. The CBA ignores potential substitution and anticipation effects. The costs to society ignore the value of leisure and potential distributional and health impacts of the measure. The CBA spans all eight years of the ATT analysis (Section 6.1). Year 1 is the first year for which we calculate the ATT. Year 8 only contains information from the 2003 sample.

In line with our employment analysis we considered the heterogeneous effects of splitting the sample by age, using the same cut-off as in the employment analysis (see Figure C.2 in Appendix C). This analysis shows that while the policy is more costly for younger workers when we do not consider replacement, it also has the greatest scope for positive budgetary effects once the replacement is taken into account. The potential benefits are greater and longer-lasting for (especially female) younger workers, as they also have longer lasting positive employment effects (cf. Section 6.1).

\section{Conclusion}

This paper studies the effect on employment of a scheme that facilitates gradual retirement through working time reductions. Recently, many EU countries have implemented such measures with the aim to postpone the retirement of older workers. However, evidence on the effectiveness of such policies is scarce and provides mixed results. Our research provides new evidence on this question by evaluating the impact of the Time Credit (TC) scheme in Belgium allowing workers above the age of 50 to reduce their working time until the statutory retirement age. 
We contributed methodologically to this literature by explicitly taking the dynamic assignment into treatment into account (Johansson and Fredriksson 2008), and by explicitly considering a competing risks model that allows measuring the treatment effect according to different (possibly selective) pathways to early retirement. To model this dynamic selection and possibly selective right censoring within this competing risks model, we slightly adjusted the methodology recently proposed by Vikström (2014) to take the stock sampling in employment at the start of the treatment into account. We find that the correction for selective right censoring matters to avoid bias, especially in the competing risks framework.

Overall our evidence is in line with the findings of Graf et al. (2011). Participation in TC initially prolongs the time spent in employment (during the first two years for men and four years for women), but subsequently it accelerates the exit to early retirement. In the beginning the effect is positive, presumably because participants are not yet eligible to early retirement. However, as soon as they are, participants have much higher incentives to enter it than non-participants, because the replacement rates in these schemes (with regard to their labour income) are much higher for TC participants than for non-participants. These financial incentives are on the one hand much stronger for individuals who reduce their working time to $50 \%$ of a full-time job than for those who reduce it to $80 \%$, and on the other hand also for workers who are eligible for the statutory early retirement scheme relative to those who are not. Our evidence supports that individuals behave according to these incentives. Besides, the impact of TC is more favourable for women than for men, because women have less opportunities to enter early retirement.

The gradual retirement scheme fails the cost-benefit test. Only under the extreme assumption that employers fully compensate for all working time reductions by new equally productive workers the TC scheme displays a net benefit for society during the first two (four) years for men (women). Nevertheless, our findings suggest that the scheme could become socially cost-effective if the opportunities for early retirement were blocked or made financially less attractive. In the analysis in which we allowed for three different exit destinations from employment, we found indeed that for the exit destinations that were financially less attractive the effect of the TC on the survival rate in employment no longer became negative or even remained significantly positive in some cases.

Given this responsiveness to the financial incentives, we believe it may be possible to prolong the positive effect of TC schemes by eliminating the perverse incentives to exit earlier from the labour force. A possible policy proposal to enhance the scheme might be to leave the entitlement to full-time pension only to the participants exiting at the statutory retirement age and accordingly adjust the entitlements of the early leavers. Since 2015 the Belgian government has raised the eligibility age to bridge pensions from 58 to 62 years (with some exceptions) and the minimum age to be eligible to the 
conventional early retirement is gradually increased since 2012 from 60 years to attain 63 years in 2019. Based on our findings we speculate that this could increase the effectiveness of the Time-Credit scheme. However, since 2015 the Belgian government has also raised the minimum age of eligibility of the end of career TC to 60 years. As a consequence, the starting age of gradual retirement has been set so close to the minimum early retirement age that it can hardly have any significant positive effect on the career length of employees. While the decision to raise the early retirement age can be supported on the basis of our findings, the decision to simultaneously increase the minimum age of eligibility to the end of career TC scheme cannot. We call for further research to obtain better foundations for these policy recommendations. 


\section{Appendix}

\section{A. Comparison of Gradual or Part-Time Retirement Schemes in Other European Countries}

Table A.1: Comparison of Gradual or Part-Time Retirement Schemes in Other European Countries

\begin{tabular}{|c|c|c|c|c|c|}
\hline Country & Policy & Years in place & Age-Eligibility & Replacement Rate & Reduction in Hours \\
\hline \multirow{2}{*}{ Sweden } & Part Time Pension & $1976-2000$ & $61 y$ & 55\% from 1994 onwards & 10h/week (i.e. max 25\% workweek) \\
\hline & Part Time Pension & $2003-\ldots$ & $61 y$ & $60 \%$ of reduction in wage & as much as $50 \%$ until $65 y$ \\
\hline Finland & Part-Time Pension & $\begin{array}{l}\text { Since } 1987 \text { in the } \\
\text { private sector, } 1989 \\
\text { public sector }\end{array}$ & $\begin{array}{l}56(<2005) \text {, then } 58 \text { and } 60 \\
(>=2011) . \text { Until } 64\end{array}$ & $\begin{array}{l}50 \% \text { difference regular and } \\
\text { part-time earnings }\end{array}$ & 16-28h/week \\
\hline Denmark & Part Time pensions & 1987 & $60-64$ & fixed rate/reduced hour & having a workweek of $12-30 \mathrm{~h} /$ week \\
\hline France & Phased Early Retirement (PRP) & $1988-2005$ & $55-65$ & top up of $30 \%$ & $40-50 \%$ \\
\hline Germany & Part Time Retirement & 1996-2009 & $55+$ & $70 \%$ top up & 50\% (blocking possible) \\
\hline Austria & $\begin{array}{l}\text { Old Age Part-Time scheme } \\
\text { (OAPT) }\end{array}$ & $2000 \ldots$ & $\begin{array}{l}>=55(\mathrm{~m}),>50(\mathrm{w})+\text { career } \\
\text { restrictions }\end{array}$ & $75 \%$ & $\begin{array}{l}40-60 \% \text {, max } 61 / 2 \text { y (blocking } \\
\text { possible) }\end{array}$ \\
\hline Netherlands & Life Course Regulation & 2006-2011 & $\begin{array}{l}\text { whole career, but can also be used } \\
\text { as part time retirement two years } \\
\text { before retirement) }\end{array}$ & own savings, $70 \%$ now & $50 \%$ \\
\hline
\end{tabular}




\section{B. The (Endogenous) Stratification of the Sample}

The population is stratified for each gender in 9 birth cohorts defined in Table B.1. The reference periods by birth cohort were chosen as to observe sufficient transitions in and out of private sector employment for both treatment and control groups determined as to evaluate the 2002 reform mentioned in the main text on the basis of a difference-in-differences strategy (Albanese and Cockx, 2015). Each of these 18 strata is subsequently endogenously stratified in five substrata:

1. The population exiting salaried employment in the private sector within the reference period; 2. The population entering salaried employment in the private sector within the reference period and not contained in substratum 1;

3. The population employed throughout the reference period as salaried worker in the private sector and earning a gross wage lower than $€ 100$ per day at the start of this period;

4. The population employed throughout the reference period as salaried worker in the private sector and earning at least $€ 100$ per day at the start of this period;

5. The population that was not employed as salaried worker in the private sector during the reference period, i.e. individuals who were out of the labour force, unemployed, self-employed or working in the public sector.

Table B.1 : Retained Birth Cohorts and Corresponding Reference Periods

\begin{tabular}{|c|c|c|}
\hline & Cohort (quarter/year) & Reference Period (quarter/year) \\
\hline 1 & $2 / 41-1 / 42$ & {$[2 / 99-1 / 02]$} \\
\hline 2 & $2 / 42-1 / 43$ & {$[2 / 99-4 / 01]$} \\
\hline 3 & $2 / 43-1 / 44$ & {$[2 / 99-4 / 03]$} \\
\hline 4 & $2 / 44-1 / 45$ & {$[2 / 00-1 / 05]$} \\
\hline 5 & $2 / 45-1 / 46$ & {$[2 / 99-4 / 03]$} \\
\hline 6 & $2 / 46-1 / 47$ & {$[2 / 00-4 / 04]$} \\
\hline 7 & $2 / 47-1 / 48$ & {$[2 / 00-3 / 05]$} \\
\hline 8 & $2 / 48-1 / 49$ & {$[2 / 02-3 / 05]$} \\
\hline 9 & $2 / 49-1 / 50$ & {$[2 / 02-3 / 05]$} \\
\hline
\end{tabular}

In each of the 18 strata a random sample of 2,000 individuals is drawn in this substratum, while the sample size was 1,500 for substratum 4 and $5 .^{37}$ The size of the population is known for each substratum, so that it was straightforward to construct the appropriate weights to make inference on the population.

\footnotetext{
${ }^{37}$ In cases that the population of the substratum was smaller than the population, the complete population was sampled.
} 


\section{Supplementary Tables and Figures}

Figure C.1: Survival Rate in Employment (Benchmark) and Competing Risks - Men (A) and Women (B)
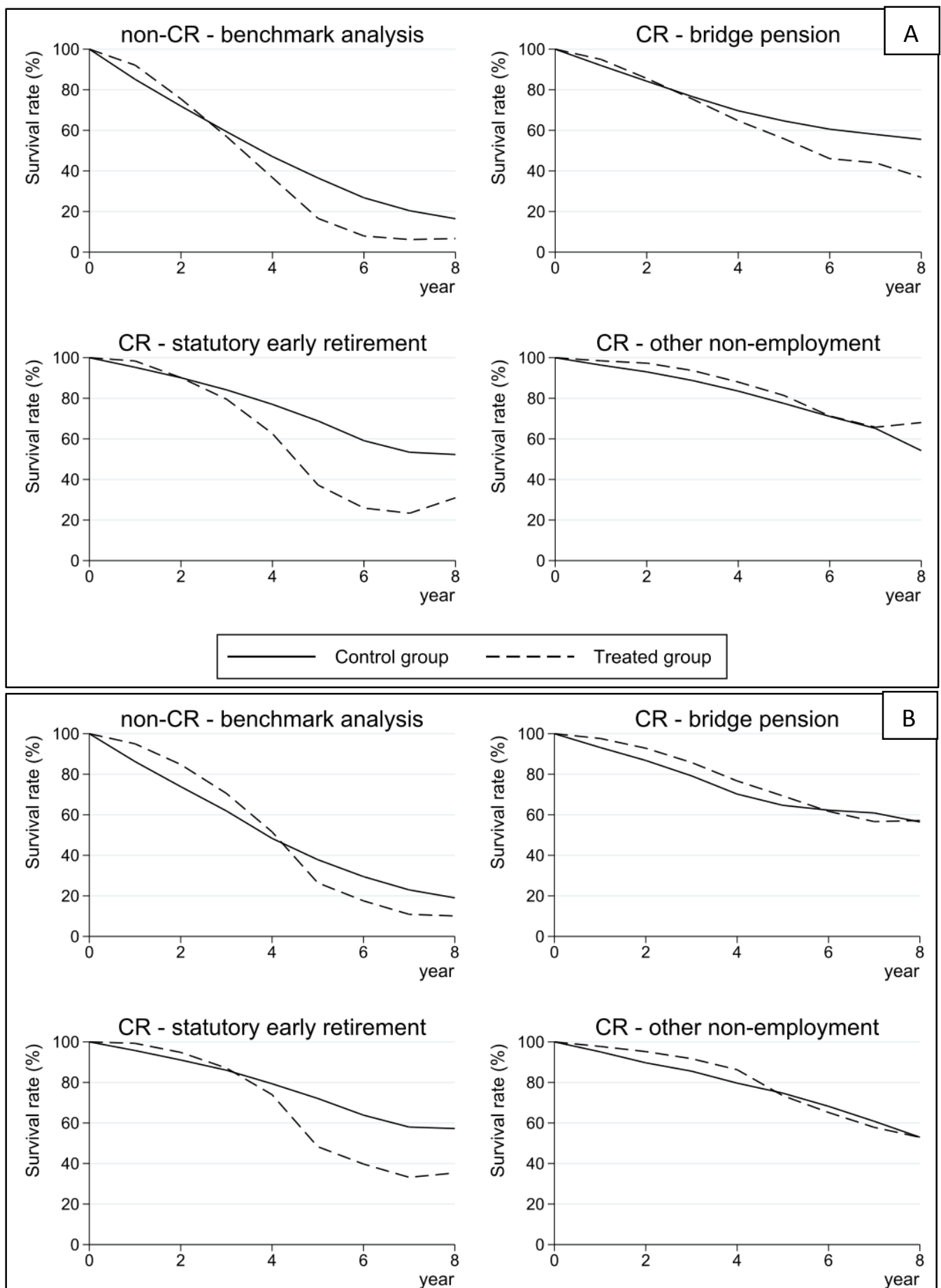

Control group

----- Treated group

Survival function of the treated and control units controlling for the dynamic selection on observables (Vikström, 2014) by gender (Panel A for men and B for women). The survival rates are expressed in percentage points (pp) and defined as (from left to right and top to bottom) (1) employment, (2) employment without exit to a bridge pension, (3) employment without exit to a statutory pension before the normal retirement age ( 65 for men and women born after 30/11/1944, 64 for women born between 01/12/1942 and 30/11/1944 and 63 for women born before 30/11//1942) and (4) employment without exit to other non-employment statuses. In the competing risk analyses (2-4), the exits from employment to other destinations, apart from the one considered, are right censored. Reported estimates are pooled over the 2003 and 2004 samples. Year eight only uses information from the 2003 sample. The pooled sample is composed of 1,227 $(762)$ treated and $29,791(9,658)$ control units (men and women). 
Figure C.2: Monthly Cost of Policy per Treated for Men (panel A) and Women (panel B)
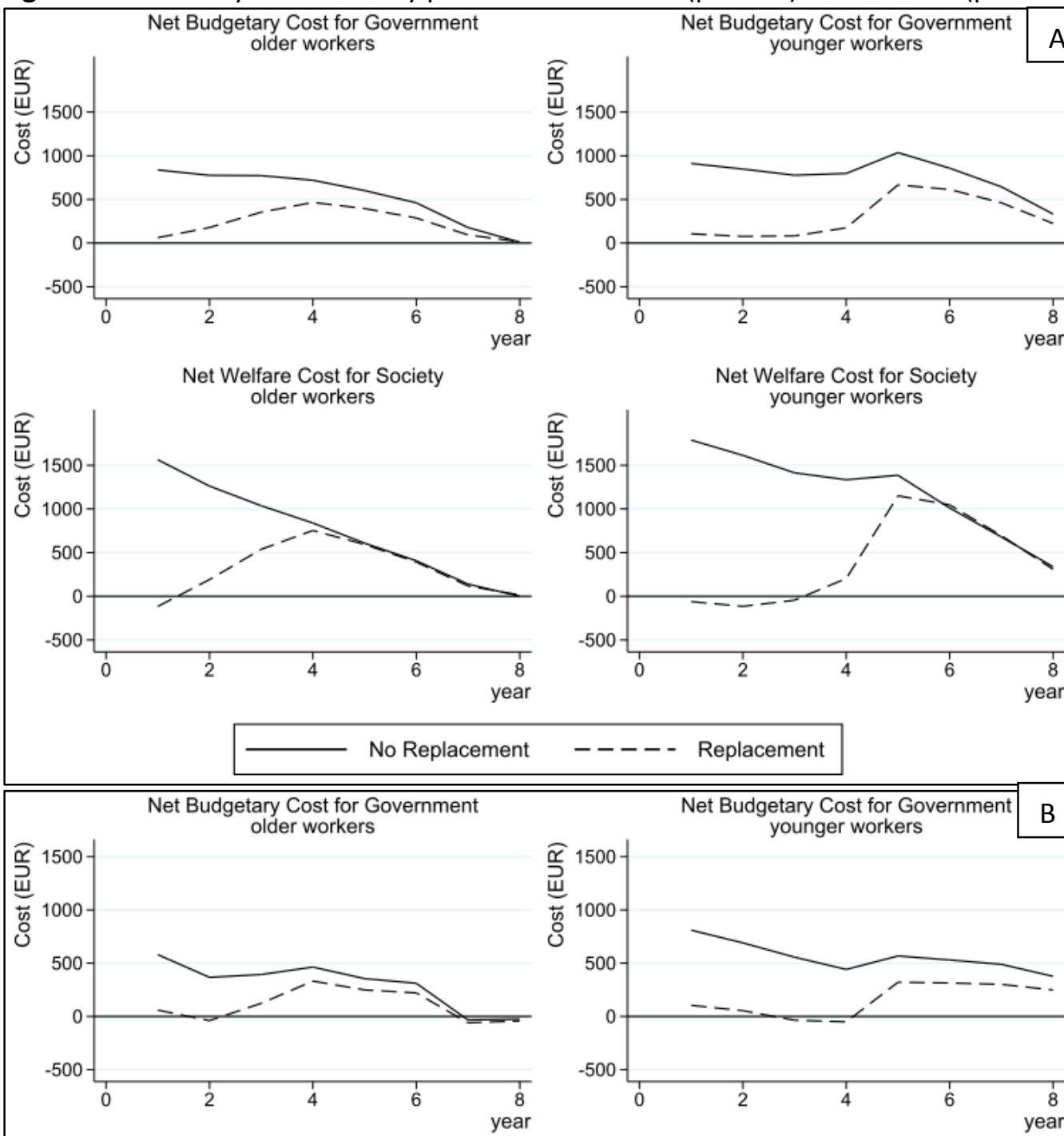

- - - Replacement

Net Welfare Cost for Society older workers
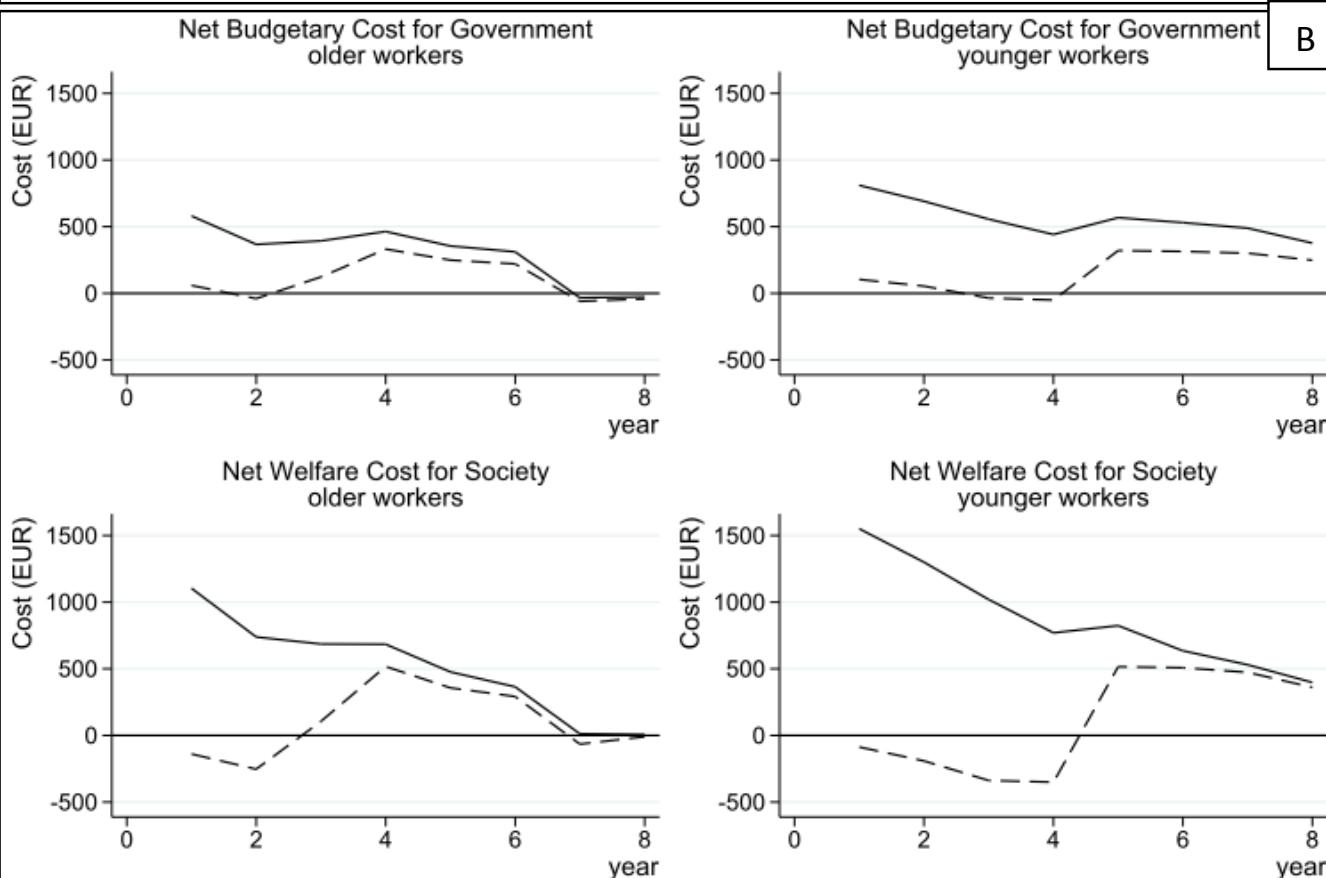

No Replacement

\section{Replacement}

Cost-benefit analysis (CBA) on the pooled sample of participants in TC of 2003 and 2004 (panel A for men, panel B for women). CBA in monthly costs (benefits if negative) in 2004 euros per treated individual (the size of the treated sample as defined in 2003 / 2004). The Net Budgetary Cost (NBC) for the government is the average cost (gain) of the policy for the state, net of savings for the public budget. The Net Welfare Cost (NWC) for society is the efficiency cost of the NBC minus the production value of employment (PV). Baseline scenario: CBA without replacement of the part-time workers. Replacement scenario: baseline scenario with additional assumption that all hours reduced by part-time workers (treated and controls) are recovered by hiring extra workers with similar characteristics. Younger workers are aged strictly below age 56.5 at the moment of sample selection (year 0), older workers are aged 56.5 and above at that moment. The CBA ignores potential substitution and anticipation effects. The costs to society ignore the value of leisure and potential distributional and health impacts of the measure. The CBA spans all eight years of the ATT analysis from Section 6.1. Year one is the first year for which we calculate the ATT. Year eight only contains information from the 2003 sample. 
Figure C.3: ATT on Survival in Employment - Heterogeneous Effects by Age

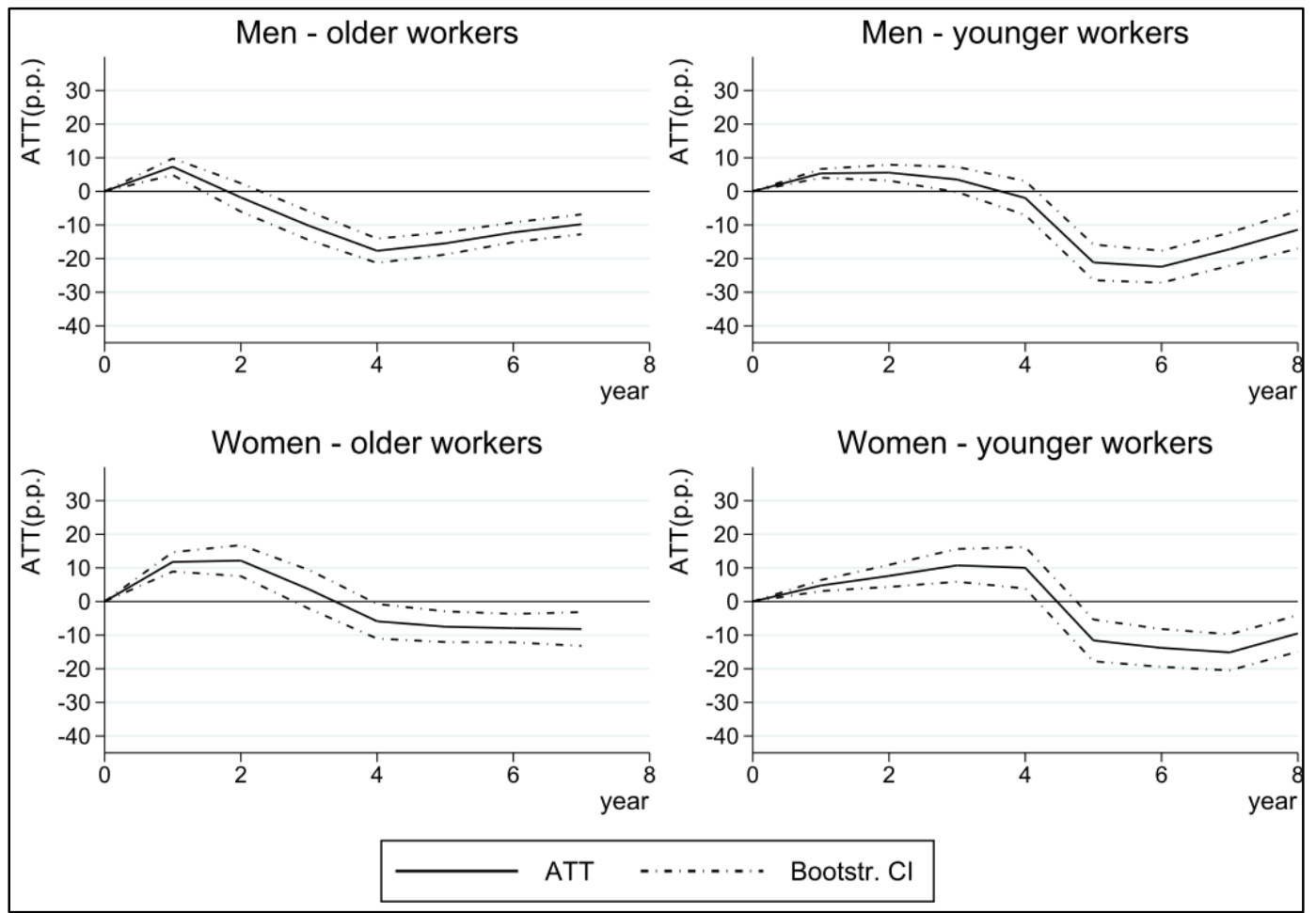

ATT on the survival rate in employment estimated by controlling for the dynamic selection on observables (Vikström, 2014). Heterogeneous effects by age in year 0: younger (below the age of 56.5) and older workers (at least 56.5 years old). The estimates of the ATT's are the percentage points (pp) differences between the survival rate of the treated in case of treatment and the estimated survival rate of the treated in the counterfactual of no treatment. Estimates are pooled over the 2003 and 2004 samples. Year eight only uses information from the 2003 sample. Standard errors are obtained by a stratified bootstrap (clustering by individual) with 500 repetitions and $95 \%$ confidence intervals (CI) by assuming normality. 
Table C.1: Sensitivity analysis on Cost-Benefit Analysis - men

\begin{tabular}{|c|c|c|c|c|c|c|c|c|c|c|c|c|c|c|c|c|c|c|}
\hline \multirow{2}{*}{$\begin{array}{l}\text { (A) } \\
\text { MCF }\end{array}$} & \multirow{2}{*}{$\begin{array}{c}\text { (B) } \\
\text { Reservation } \\
\text { Wage }\end{array}$} & \multirow{2}{*}{$\begin{array}{c}\text { (C) } \\
\text { Income } \\
\text { taxes }\end{array}$} & \multicolumn{8}{|c|}{ (1) No replacement of part-time workers } & \multicolumn{8}{|c|}{ (2) Replacement of part-time workers } \\
\hline & & & Year 1 & Year 2 & Year 3 & Year 4 & Year 5 & Year 6 & Year 7 & Year 8 & Year 1 & Year 2 & Year 3 & Year 4 & Year 5 & Year 6 & Year 7 & Year 8 \\
\hline \multicolumn{19}{|c|}{ ( $\alpha$ ) NET BUDGETARY COST (NBC) FOR THE GOVERNMENT } \\
\hline - & - & Medium & 878.3 & 803.0 & 791.2 & 817.0 & 906.1 & 732.6 & 540.0 & 388.6 & 90.7 & 119.2 & 222.7 & 359.6 & 596.2 & 508.9 & 371.5 & 273.8 \\
\hline- & - & High & 914.1 & 835.7 & 823.1 & 850.5 & 941.4 & 760.2 & 559.5 & 402.6 & 78.6 & 111.5 & 224.3 & 373.8 & 627.5 & 537.0 & 391.3 & 288.0 \\
\hline \multicolumn{19}{|c|}{ ( $\beta$ ) NET WELFARE COST (NWC) FOR SOCIETY } \\
\hline 1.41 & Low & Medium & $1,505.2$ & $1,306.4$ & $1,185.0$ & $1,142.1$ & $1,123.5$ & 817.1 & 530.7 & 371.0 & -306.8 & -170.3 & 126.0 & 494.0 & 992.0 & 839.2 & 544.8 & 373.1 \\
\hline 1.41 & Low & High & $1,519.8$ & $1,319.8$ & $1,198.1$ & $1,155.9$ & $1,138.0$ & 828.4 & 538.7 & 376.7 & -311.8 & -173.5 & 126.7 & 499.8 & $1,004.8$ & 850.7 & 552.9 & 378.9 \\
\hline 1.41 & Medium & Medium & $1,022.1$ & 864.3 & 755.4 & 698.0 & 653.1 & 450.9 & 271.5 & 182.8 & -143.7 & -67.9 & 100.0 & 305.7 & 575.4 & 464.8 & 279.5 & 182.1 \\
\hline 1.41 & Medium & High & $1,054.7$ & 894.1 & 784.4 & 728.4 & 685.3 & 475.9 & 289.2 & 195.5 & -154.7 & -74.9 & 101.5 & 318.7 & 603.9 & 490.4 & 297.6 & 195.0 \\
\hline 1.41 & High & Medium & 539.1 & 422.1 & 325.8 & 253.8 & 182.6 & 84.6 & 12.4 & -5.5 & 19.3 & 34.6 & 74.0 & 117.4 & 158.9 & 90.5 & 14.2 & -8.9 \\
\hline 1.41 & High & High & 589.6 & 468.3 & 370.7 & 300.9 & 232.5 & 123.4 & 39.7 & 14.3 & 2.3 & 23.6 & 76.3 & 137.5 & 203.0 & 130.1 & 42.2 & 11.0 \\
\hline 2.14 & Low & Medium & $2,189.4$ & $1,906.3$ & $1,723.1$ & $1,644.4$ & $1,602.2$ & $1,176.1$ & 786.7 & 560.6 & -243.5 & -85.9 & 285.4 & 753.1 & $1,421.1$ & $1,206.5$ & 807.4 & 566.6 \\
\hline 2.14 & Low & High & $2,230.2$ & $1,943.6$ & $1,759.5$ & $1,682.5$ & $1,642.6$ & $1,207.6$ & 808.8 & 576.5 & -257.3 & -94.7 & 287.3 & 769.4 & $1,456.8$ & $1,238.6$ & 830.0 & 582.8 \\
\hline 2.14 & Medium & Medium & $1,706.4$ & $1,464.1$ & $1,293.5$ & $1,200.2$ & $1,131.8$ & 809.9 & 527.5 & 372.3 & -80.4 & 16.5 & 259.4 & 564.9 & $1,004.6$ & 832.2 & 542.1 & 375.6 \\
\hline 2.14 & Medium & High & $1,765.1$ & $1,517.8$ & $1,345.7$ & $1,255.1$ & $1,189.8$ & 855.1 & 559.3 & 395.3 & -100.3 & 3.8 & 262.1 & 588.3 & $1,055.9$ & 878.3 & 574.6 & 398.8 \\
\hline 2.14 & High & Medium & $1,223.3$ & $1,022.0$ & 863.9 & 756.0 & 661.4 & 443.6 & 268.3 & 184.1 & 82.6 & 119.0 & 233.4 & 376.6 & 588.0 & 457.9 & 276.8 & 184.7 \\
\hline 2.14 & High & High & $1,299.9$ & $1,092.1$ & 932.0 & 827.6 & 737.1 & 502.6 & 309.9 & 214.1 & 56.8 & 102.4 & 236.9 & 407.1 & 654.9 & 518.1 & 319.3 & 214.9 \\
\hline 3.23 & Low & Medium & $3,211.1$ & $2,801.9$ & $2,526.6$ & $2,394.4$ & $2,317.1$ & $1,712.2$ & $1,168.9$ & 843.7 & -149.0 & 40.2 & 523.5 & $1,140.1$ & $2,061.9$ & $1,755.1$ & $1,199.4$ & 855.7 \\
\hline 3.23 & Low & High & $3,290.9$ & $2,874.9$ & $2,597.6$ & $2,469.0$ & $2,396.0$ & $1,773.6$ & $1,212.2$ & 874.9 & -176.0 & 22.9 & 527.0 & $1,171.9$ & $2,131.6$ & $1,817.8$ & $1,243.7$ & 887.2 \\
\hline 3.23 & Medium & Medium & $2,728.0$ & $2,359.8$ & $2,097.0$ & $1,950.2$ & $1,846.6$ & $1,345.9$ & 909.7 & 655.4 & 14.1 & 142.6 & 497.5 & 951.9 & $1,645.3$ & $1,380.8$ & 934.1 & 664.7 \\
\hline 3.23 & Medium & High & $2,825.8$ & $2,449.2$ & $2,183.9$ & $2,041.5$ & $1,943.2$ & $1,421.1$ & 962.7 & 693.6 & -18.9 & 121.4 & 501.8 & 990.8 & $1,730.7$ & $1,457.5$ & 988.4 & 703.3 \\
\hline 3.23 & High & Medium & $2,245.0$ & $1,917.6$ & $1,667.3$ & $1,506.0$ & $1,376.2$ & 979.7 & 650.5 & 467.2 & 177.2 & 245.0 & 471.5 & 763.6 & $1,228.8$ & $1,006.4$ & 668.8 & 473.7 \\
\hline 3.23 & High & High & $2,360.6$ & $2,023.4$ & $1,770.2$ & $1,614.1$ & $1,490.5$ & $1,068.7$ & 713.2 & 512.4 & 138.1 & 220.0 & 476.7 & 809.7 & $1,329.8$ & $1,097.3$ & 733.0 & 519.4 \\
\hline
\end{tabular}

Cost-benefit analysis (CBA) on the pooled sample of male participants in TC of 2003 and 2004. Treated sample size defined in 2003-2004. CBA in monthly costs (benefits if negative) in 2004 euros per treated individual under different scenarios. Scenario in bold denotes baseline scenario. (1) No replacement of part-time workers scenario; and (2) replacement scenario: baseline scenario with additional assumption that all hours reduced by part-time workers (treated and controls) are recovered by hiring extra workers with similar characteristics. Additionally (A) Marginal Cost of public Funds (MCF) equal to 1.41, 2.14, or 3.23 (Kleven and Kreiner, 2006), (B) opportunity cost of working (Reservation Wage) with a lower, medium and upper bound (see Section 7.1) and (C) Income Tax Rate, variable over time: medium (average income) which is on average $28.26 \%$, higher (133\% average income) which is on average $32.36 \%$ (OECD stat extract, 2003-2011). The first outcome variable is the Net Budgetary Cost (NBC) for the government ( $\alpha$ ), i.e. the average cost (gain) of the policy for the state, net of savings for the public budget. The second is the Net Welfare Cost (NWC) for society ( $\beta$ ), i.e. the efficiency cost of the NBC minus the production value of employment. The CBA ignores potential substitution and anticipation effects. The costs to society ignore potential distributional and health impacts of the measure. The CBA spans all eight years of the ATT analysis from Section 6.1. Year one is the first year for which we calculate the ATT. Year eight only contains information from the 2003 sample 
Table C.2: Sensitivity analysis on Cost-Benefit Analysis - women

\begin{tabular}{|c|c|c|c|c|c|c|c|c|c|c|c|c|c|c|c|c|c|c|}
\hline \multirow{2}{*}{$\begin{array}{l}\text { (A) } \\
\text { MCF }\end{array}$} & \multirow{2}{*}{$\begin{array}{c}\text { (B) } \\
\text { Reservation } \\
\text { Wage }\end{array}$} & \multirow{2}{*}{$\begin{array}{c}\text { (C) } \\
\text { Income } \\
\text { taxes }\end{array}$} & \multicolumn{8}{|c|}{ (1) No replacement of part-time workers } & \multicolumn{8}{|c|}{ (2) Replacement of part-time workers } \\
\hline & & & Year 1 & Year 2 & Year 3 & Year 4 & Year 5 & Year 6 & Year 7 & Year 8 & Year 1 & Year 2 & Year 3 & Year 4 & Year 5 & Year 6 & Year 7 & Year 8 \\
\hline \multicolumn{19}{|c|}{ ( $\alpha$ ) NET BUDGETARY COST (NBC) FOR THE GOVERNMENT } \\
\hline- & - & Medium & 695.5 & 533.5 & 485.0 & 466.0 & 565.1 & 495.5 & 429.6 & 357.6 & 67.0 & 3.8 & 33.5 & 119.3 & 362.8 & 322.8 & 271.7 & 237.8 \\
\hline- & - & High & 721.9 & 554.2 & 504.2 & 484.8 & 589.2 & 515.5 & 446.2 & 371.4 & 53.9 & -11.5 & 22.3 & 115.9 & 379.7 & 339.2 & 286.0 & 250.7 \\
\hline \multicolumn{19}{|c|}{ ( $\beta$ ) NET WELFARE COST (NWC) FOR SOCIETY } \\
\hline 1.41 & Low & Medium & $1,139.7$ & 872.2 & 761.1 & 684.7 & 777.2 & 598.3 & 467.6 & 374.5 & -366.8 & -442.7 & -302.2 & -50.5 & 558.5 & 496.6 & 414.6 & 355.8 \\
\hline 1.41 & Low & High & $1,150.6$ & 880.8 & 769.0 & 692.4 & 787.1 & 606.5 & 474.4 & 380.2 & -372.2 & -449.0 & -306.8 & -51.9 & 565.4 & 503.3 & 420.5 & 361.1 \\
\hline 1.41 & Medium & Medium & 790.1 & 601.2 & 511.0 & 439.7 & 461.9 & 335.8 & 248.9 & 192.9 & -185.7 & -228.9 & -145.7 & -2.8 & 336.2 & 281.3 & 224.9 & 186.6 \\
\hline 1.41 & Medium & High & 814.2 & 620.1 & 528.5 & 456.8 & 483.8 & 354.1 & 264.0 & 205.5 & -197.6 & -242.8 & -155.8 & -5.9 & 351.5 & 296.3 & 238.0 & 198.3 \\
\hline 1.41 & High & Medium & 440.5 & 330.1 & 261.0 & 194.7 & 146.6 & 73.3 & 30.1 & 11.3 & -4.6 & -15.0 & 10.9 & 44.9 & 113.9 & 66.1 & 35.1 & 17.3 \\
\hline 1.41 & High & High & 477.8 & 359.4 & 288.0 & 221.2 & 180.5 & 101.6 & 53.5 & 30.8 & -23.1 & -36.6 & -4.9 & 40.1 & 137.7 & 89.2 & 55.4 & 35.5 \\
\hline 2.14 & Low & Medium & $1,694.9$ & $1,324.0$ & $1,156.3$ & $1,032.4$ & $1,125.8$ & 876.3 & 692.4 & 559.0 & -320.9 & -445.0 & -286.3 & 25.1 & 819.6 & 730.0 & 611.2 & 527.5 \\
\hline 2.14 & Low & High & $1,725.0$ & $1,347.7$ & $1,178.2$ & $1,053.8$ & $1,153.2$ & 899.2 & 711.3 & 574.7 & -335.8 & -462.5 & -299.1 & 21.3 & 838.9 & 748.7 & 627.6 & 542.2 \\
\hline 2.14 & Medium & Medium & $1,345.3$ & $1,052.9$ & 906.2 & 787.5 & 810.5 & 613.8 & 473.6 & 377.4 & -139.8 & -231.1 & -129.8 & 72.9 & 597.3 & 514.7 & 421.4 & 358.3 \\
\hline 2.14 & Medium & High & $1,388.6$ & $1,087.0$ & 937.7 & 818.2 & 849.9 & 646.7 & 500.8 & 400.0 & -161.3 & -256.3 & -148.2 & 67.3 & 625.0 & 541.6 & 445.0 & 379.4 \\
\hline 2.14 & High & Medium & 995.7 & 781.8 & 656.2 & 542.5 & 495.1 & 351.4 & 254.8 & 195.8 & 41.3 & -17.3 & 26.7 & 120.6 & 375.0 & 299.5 & 231.6 & 189.1 \\
\hline 2.14 & High & High & $1,052.3$ & 826.4 & 697.3 & 582.7 & 546.6 & 394.3 & 290.4 & 225.3 & 13.3 & -50.1 & 2.8 & 113.3 & 411.2 & 334.6 & 262.4 & 216.7 \\
\hline 3.23 & Low & Medium & $2,523.8$ & $1,998.5$ & $1,746.4$ & $1,551.7$ & $1,646.3$ & $1,291.5$ & $1,027.9$ & 834.5 & -252.3 & -448.4 & -262.7 & 138.1 & $1,209.5$ & $1,078.5$ & 904.6 & 784.0 \\
\hline 3.23 & Low & High & $2,582.7$ & $2,044.9$ & $1,789.2$ & $1,593.6$ & $1,699.9$ & $1,336.2$ & $1,065.0$ & 865.2 & -281.6 & -482.6 & -287.6 & 130.6 & $1,247.2$ & $1,115.0$ & 936.7 & 812.7 \\
\hline 3.23 & Medium & Medium & $2,174.2$ & $1,727.4$ & $1,496.3$ & $1,306.8$ & $1,330.9$ & $1,029.0$ & 809.2 & 652.9 & -71.2 & -234.5 & -106.1 & 185.8 & 987.2 & 863.2 & 714.8 & 614.8 \\
\hline 3.23 & Medium & High & $2,246.3$ & $1,784.2$ & $1,548.8$ & $1,358.0$ & $1,396.6$ & $1,083.7$ & 854.5 & 690.5 & -107.0 & -276.4 & -136.7 & 176.6 & $1,033.3$ & 908.0 & 754.1 & 649.9 \\
\hline 3.23 & High & Medium & $1,824.6$ & $1,456.4$ & $1,246.3$ & $1,061.8$ & $1,015.6$ & 766.5 & 590.4 & 471.3 & 109.9 & -20.7 & 50.4 & 233.5 & 765.0 & 648.0 & 525.1 & 445.6 \\
\hline 3.23 & High & High & $1,910.0$ & $1,523.5$ & $1,308.3$ & $1,122.4$ & $1,093.3$ & 831.3 & 644.1 & 515.8 & 67.5 & -70.2 & 14.3 & 222.7 & 819.5 & 700.9 & 571.5 & 487.2 \\
\hline
\end{tabular}

Cost-benefit analysis (CBA) on the pooled sample of female participants in TC of 2003 and 2004. Treated sample size defined in 2003-2004. CBA in monthly costs (benefits if negative) in 2004 euros per treated individual under different scenarios. Scenario in bold denotes baseline scenario. (1) No replacement of part-time workers scenario; and (2) replacement scenario: baseline scenario with additional assumption that all hours reduced by part-time workers (treated and controls) are recovered by hiring extra workers with similar characteristics. Additionally (A) Marginal Cost of public Funds (MCF) equal to 1.41, 2.14, or 3.23 (Kleven and Kreiner, 2006), (B) opportunity cost of working (Reservation Wage) with a lower, medium and upper bound (see Section 7.1) and (C) Income Tax Rate, variable over time: medium (income tax on the average income) which is on average $28.26 \%$, higher (income tax on $133 \%$ of the average income) which is on average $32.36 \%$ (OECD stat extract, 2003-2011). The first outcome variable is the Net Budgetary Cost (NBC) for the government ( $\alpha$ ), i.e. the average cost (gain) of the policy for the state, net of savings for the public budget. The second is the Net Welfare Cost (NWC) for society ( $\beta$ ), i.e. the efficiency cost of the NBC minus the production value of employment. The CBA ignores potential substitution and anticipation effects. The costs to society ignore potential distributional and health impacts of the measure. The CBA spans all eight years of the ATT analysis from Section 6.1. Year one is the first year for which we calculate the ATT. Year eight only contains information from the 2003 sample. 
Table C.3 : Balancing tests: Standardized Bias (SB), pvalue on mean equality and others

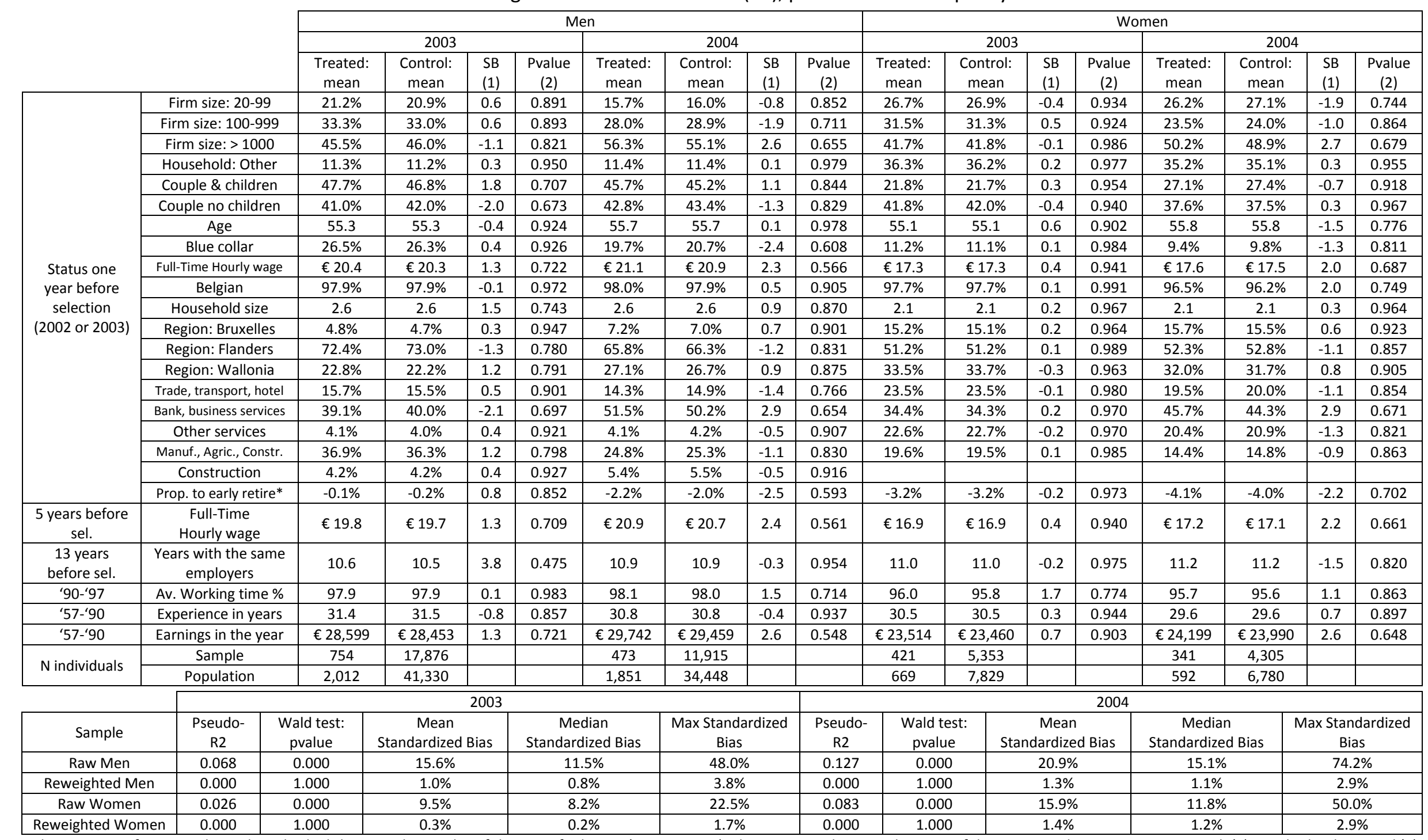

Balancing tests after reweighting the individuals by using the weights of the year of selection (2003 or 2004). The upper panel reports the mean of the covariates by treatment status with (1) Standardized Bias and (2) pvalue of the t-test. The lower panel shows the results of other balancing tests before and after reweighting the individuals by such weights. 


\section{Bibliography}

Ahn, T., 2015. Reduction of Working Time: Does It Lead to a Healthy Lifestyle? Forthcoming in Health Economics.

Albanese, A., Cockx, B., 2015. Permanent Wage Cost Subsidies for Older Workers: An Effective Tool for Increasing Working Time and Postponing Early Retirement? IZA Discussion Paper No. 8988, IZA, Bonn.

Aubert, P., Crépon, B., 2003. La productivité des salariés âgés: une tentative d'estimation. Économie et statistique 368(1), 95-119.

Aubert, P., Crépon, B., 2006. Are older workers less productive? Firm-level evidence on ageproductivity and age-wage profiles. Mimeo, INSEE, Paris.

Barrios Cobos, S., Saveyn, B., Pycroft, J., 2013. The Marginal Cost of Public Funds in the Eu: The Case of Labour Versus Green Taxes. Taxation papers 35, Publications Office of the European Union.

Berg, P.B., Hamman, M.K., Piszczek, M., Ruhm, C., 2015. Can Policy Facilitate Partial Retirement? Evidence from Germany . IZA Discussion Paper No. 9266, IZA, Bonn.

Busso, M., DiNardo, J., McCrary, J., 2014. New Evidence on the Finite Sample Properties of Propensity Score Reweighting and Matching Estimators. The Review of Economics and Statistics 96(5), 885-897.

Cameron, A.C., Trivedi, P.K., 2005. Microeconometrics: methods and applications. Cambridge University Press.

Cardoso, A.R., Guimarães, P., Varejão, J., 2011. Are Older Workers Worthy of Their Pay? An Empirical Investigation of Age-Productivity and Age-Wage Nexuses. De Economist 159(2), 95-111.

Cataldi, A., Kampelmann, S., Rycx, F., 2012. Does it pay to be productive? The case of age groups. International Journal of Manpower 33, 264-283.

Charles, K., Decicca, P., 2007. Hours Flexibility and Retirement. Economic Inquiry 45(2), 251-267.

Crépon, B., Ferracci, M., Jolivet, G., van den Berg, G., 2009. Active Labor Market Policy Effects in a Dynamic Setting. Journal of the European Economic Association 7(2-3), 595-605.

Devisscher, S., 2004. The Career Break (Time Credit) Scheme in Belgium and the Incentive Premiums by the Flemish Government. Discussion Paper, IDEA Consult, Brussels.

Devisscher, S., Sanders, D., 2007. Ageing and Life-course Issues: The Case of the Career Break Scheme (Belgium) and the Life-course Regulation (Netherlands). In OECD, Modernising Social Policy for the New Life Course (pp. 117-132). 117-132.

Elsayed, A., A., de Grip, D., Fouarge, R., Montizaan, 2015. Gradual Retirement, Financial Incentives, and Labour Supply of Older Workers: Evidence from a Stated Preference Analysis. IZA Discussion Paper No. 9430, IZA, Bonn.

Eurofound, 2001. Progressive Retirement in Europe. European Observatory of Working Life . Dublin. http://www.eurofound.europa.eu/observatories/eurwork/comparativeinformation/progressive-retirement-in-europe.

Fredriksson, P., Johansson, P., 2008. Dynamic Treatment Assignment: The Consequences for Evaluations Using Observational Data. Journal of Business \& Economic Statistics 26(4), 435445.

Frimmel, W., Horvath, T., Schnalzenberger, M., Winter-Ebmer, R., 2015. Seniority Wages and the Role of Firms in Retirement. IZA Discussion Paper No. 9192, IZA, Bonn.

Frölich, M., 2007. Propensity Score Matching Without Conditional Independence Assumption-with an Application to the Gender Wage Gap in the United Kingdom. The Econometrics Journal 10(2), 359-407.

Frölich, M., Huber, M., Wiesenfarth, M., 2015. The Finite Sample Performance of Semi- and Nonparametric Estimators for Treatment Effects and Policy Evaluation . IZA Discussion Papers No. 8756, IZA, Bonn. 
Gielen, A., 2009. Working hours flexibility and older workers' labor supply. Oxford Economic Papers, Oxford University Press 61(2), 240-274.

Graf, N., Hofer, H., Winter-Ebmer, R., 2011. Labor supply effects of a subsidized old-age part-time scheme in Austria. Zeitschrift für ArbeitsmarktForschung - Journal for Labour Market Research 44(3), 217-229.

Greenberg, D., Robins, P., 2008. Incorporating nonmarket time into benefit-cost analyses of social programs: An application to the self-sufficiency project. Journal of Public Economics 92(3-4), 766-794.

Gustman, A., Steinmeier, T., 1984. Partial Retirement and the Analysis of Retirement Behavior. Industrial and Labor Relations Review 37(3), 403-415.

Hellerstein, J.K., Neumark, D., 2007. Production function and wage equation estimation with heterogeneous labor: Evidence from a new matched employer-employee data set, in: Hardto-Measure Goods and Services: Essays in Honor of Zvi Griliches. University of Chicago Press, pp. 31-71.

Hellerstein, J., Neumark, D., Troske, K., 1999. Wages, Productivity, and Worker Characteristics: Evidence from Plant-Level Production Functions and Wage Equations. Journal of Labor Economics 17(3), 409-46.

Hirano, K., Imbens, G.W., Ridder, G., 2003. Efficient Estimation of Average Treatment Effects Using the Estimated Propensity Score. Econometrica 71(4), 1161-1189.

Honig, M., Hanoch, G., 1985. Partial Retirement as a Separate Mode of Retirement Behavior. The Journal of Human Resources 20(1), 21-46.

Horvitz, D.G., Thompson, D.J., 1952. A Generalization of Sampling Without Replacement from a Finite Universe. Journal of the American Statistical Association 47(260), 663-685.

Huber, M., Lechner, M., Wunsch, C., 2013. The Performance of Estimators Based on the Propensity Score. Journal of Econometrics 175(1), 1-21.

Huber, M., Lechner, M., Wunsch, C., 2015. The effect of firms' phased retirement policies on the labour market outcomes of their employees. Forthcoming in the Industrial and Labor Relations Review.

Hurd, M.D., 1996. The Effect of Labor Market Rigidities on the Labor Force Behavior of Older Workers. In: Wise D, editor. Advances in the Economics of Aging. University of Chicago Press 11-60, Chicago and London.

Hutchens, R., 2010. Worker characteristics, job characteristics, and opportunities for phased retirement. Labour Economics 17(6), 1010-1021.

Hutchens, R., Grace-Martin, K., 2006. Employer Willingness to Permit Phased Retirement: Why are Some More Willing Than others? ILR Review 59(4), 525-546.

Ilmakunnas, P., Maliranta, M., 2005. Technology, Labour Characteristics and Wage-productivity Gaps. Oxford Bulletin of Economics and Statistics 67(5), 623-645.

Imbens, G., Wooldridge, J., 2009. Recent Developments in the Econometrics of Program Evaluation. Journal of Economic Literature 47(1), 5-86.

Kantarcı, T., van Soest, A., 2008. Gradual Retirement: Preferences and Limitations. De Economist 156(2), 113-144.

Kleven, H.J., Kreiner, C., 2006. The Marginal Cost of Public Funds: Hours of Work Versus Labor Force Participation. Journal of Public Economics 90(10-11), 1955-1973.

Lazear, E., 1979. Why Is There Mandatory Retirement? Journal of Political Economy 87(6), 1261-84.

Lechner, M., Miquel, R., Wunsch, C., 2011. Long-Run Effects of Public Sector Sponsored Training in West Germany. Journal of the European Economic Association 9(4), 742-784.

Lechner, M., Strittmatter, A., 2014. Practical Procedures to Deal with Common Support Problems in Matching Estimation . Economics Working Paper Series No. 1410, University of St. Gallen, St. Gallen.

Lechner, M., Wunsch, C., 2008. What Did All the Money Do? On the General Ineffectiveness of Recent West German Labour Market Programmes. Kyklos 61(1), 134-174. 
Machado, C.S., Portela, M., 2012. Hours of Work and Retirement Behavior . IZA Discussion Paper No. 6270, IZA, Bonn.

Manski, C., Lerman, S.R., 1977. The Estimation of Choice Probabilities from Choice Based Samples. Econometrica 45(8), 1977-88.

OECD, 2015a. OECD.StatExtracts. Retrieved February 4, 2015, from www.stats.oecd.org.

OECD, 2015b. Ageing and Employment Policies - Statistics on average effective age of retirement.

Rudolf, R., 2014. Work Shorter, Be Happier? Longitudinal Evidence from the Korean Five-Day Working Policy. Journal of Happiness Studies 15(5), 1139-1163.

Ruhm, C., 1990. Bridge Jobs and Partial Retirement. Journal of Labor Economics 8(4), 482-501.

Schmid, G., 1998. Transitional labour markets: A new European employment strategy. WZB Discussion Paper, No. FS I 98-206.

Shao, J., 2003. Impact of the Bootstrap on Sample Surveys. Statistical Science 18(2), 191-198.

Sianesi, B., 2004. An Evaluation of the Swedish System of Active Labor Market Programs in the 1990s. The Review of Economics and Statistics 86(1), 133-155.

Staubli, S., Zweimüller, J., 2013. Does raising the early retirement age increase employment of older workers? Journal of Public Economics 108(C), 17-32.

Sundén, A., 1994. Early Retirement in the Swedish Pension System. PhD thesis, Ithaca: Cornell University.

Vandenberghe, V., Waltenberg, F., Rigo, M., 2013. Ageing and Employability. Evidence from Belgian Firm Level Data. Journal of Productivity Analysis 40, 111-136.

Van Looy, P., Kovalenko, M., Mortelmans, D., De Preter, H., 2014. Working hours-reduction in the move to full retirement: How does this affect retirement preferences of $50+$ individuals in Flanders? Leuven: Steunpunt WSE/Antwerpen: CELLO, Universiteit Antwerpen.

van Ours, J., Stoeldraijer, L., 2011. Age, Wage and Productivity in Dutch Manufacturing. De Economist 159(2), 113-137.

Vikström, J., 2014. IPW estimation and related estimators for evaluation of active labor market policies in a dynamic setting. Working Paper Series from IFAU - Institute for Evaluation of Labour Market and Education Policy, No 2014:1, Uppsala.

Wadensjö, E., 2006. Part-Time Pensions and Part-Time Work in Sweden . IZA Discussion Paper No. 2273, IZA, Bonn. 